\title{
Simple Non-Iterative Calibration for Triaxial Accelerometers
}

\author{
Niklas Grip* ${ }^{\ddagger}$ and Natalia Sabourova ${ }^{\ddagger}$ \\ Luleå University of Technology, SE-971 87 Luleå, Sweden, Niklas.Grip@ltu.se Natalia.Sabourova@ltu.se
}

October 22, 2011

\begin{abstract}
For high precision measurements, accelerometers need recalibration between different measurement occasions. In this paper we derive a simple calibration method for triaxial accelerometers with orthogonal axes. Just like previously proposed iterative methods, we compute the calibration parameters (biases and gains) from measurements of the Earth gravity for six different unknown orientations of the accelerometer. However, our method is non-iterative, so there are no complicated convergence issues depending on input parameters, round-off errors etc.

The main advantages of our method are that only from the accelerometer output voltages it gives a complete knowledge of whether it is possible, with any method, to recover the accelerometer biases and gains from the output voltages, and when this is possible, we have a simple explicit formula for computing them with a smaller number of arithmetic operations than in previous iterative approaches. Moreover, we show that such successful recovery is guaranteed if the six calibration measurements deviate with angles smaller than some upper bound from a natural setup with two horizontal axes. We provide an estimate from below of this upper bound that, for instance, allows 5 degree deviations in arbitrary directions for the Colibrys SF3000L accelerometers in our lab. Similar robustness is also confirmed for even larger angles in Monte Carlo simulations of both our basic method and two different least square error extensions of it for more than six measurements. These simulations compare the sensitivities to noise and crossaxis interference. For instance, for $0.5 \%$ cross-axis interference the basic method with six measurements, each with two horizontal axes, gave higher accuracy than allowing 10 degree deviation from horizontality and compensating with more measurements and least squares fitting.
\end{abstract}

Keywords: In-field calibration, non-iterative, triaxial accelerometer, orthogonal axes, gain factor, bias, offset, Colibrys SF3000L

\section{Introduction}

For many different applications each output voltage of a triaxial accelerometer can be well modeled as depending linearly on the measured acceleration via a multiplicative gain factor and a

\footnotetext{
${ }^{*}$ The author was partially supported by the Swedish Research Council (project registration number 2004-3862).

${ }^{\ddagger}$ Both authors were supported by the Swedish Research Council Formas (registration number 2007-1430).
} 
zero offset (bias). Both the gain and the bias change noticeably, for example, with the temperature 11. They can differ by $\pm 10 \%$ from one accelerometer to the next, or between the axes of the same accelerometer ${ }^{\mathbb{1}}$. This gives an error both in the direction and the size of the measured acceleration. For good reliability, for instance at different temperatures, and in collaborative use of several accelerometers, it is therefore important to calibrate the accelerometers either before measuring or repeatedly during long-time measurements.

This paper is intended for in-field calibration of triaxial accelerometers with no other sensors available for assisting the calibration. A commonly used in-field approach 2, 3, 4, 5, 6, is to assume the accelerometer to measure in three perfectly orthogonal directions and to compute the three gain and three bias parameters from measurements of the local Earth gravity for six different orientations of the accelerometer. This approach requires no explicit knowledge of the actual orientation of the accelerometer with respect to gravity.

For solving the resulting system of six nonlinear equations, different iterative numerical solution methods have been proposed 2, 4, 5, 6. A linear minimum variance unbiased estimator approach has also been suggested for some slightly different applications [3, 7. For iterative methods, more work is needed to clarify for which measurement setups there is a unique solution and whether the iterative algorithm converges to this solution. We eliminate this problem by deriving a simple non-iterative calibration method for which we can state explicit conditions on the measurement setups that guarantee successful calibration. In a direct comparison of our solution with the fast converging method proposed in [6], we show that the latter actually always converges in at most two iterations.

We derive our calibration method for accelerometers that measure in three perfectly orthogonal directions with unknown gains $g_{m}^{\text {true }}$ and biases $b_{m}^{\text {true }}$ for $m=1,2,3$. We assume, contrary to [3, 7, that the accelerometer can be rotated and placed at rest in six different angular orientations. For each measured acceleration $A_{m, n}$, we will consider the corresponding dimensionless quantity

$$
\mathcal{A}_{m, n} \stackrel{\text { def }}{=} A_{m, n} / G, \quad \text { with } G \text { being the local Earth gravity. }
$$

This gives the fundamental relation

$$
\mathcal{A}_{1, n}^{2}+\mathcal{A}_{2, n}^{2}+\mathcal{A}_{3, n}^{2}=1, \quad n=1,2, \ldots, 6 .
$$

The corresponding accelerometer voltage outputs can be organized into the matrix

$$
V=\left(\begin{array}{lll}
V_{1,1} & \cdots & V_{1,6} \\
V_{2,1} & \cdots & V_{2,6} \\
V_{3,1} & \cdots & V_{3,6}
\end{array}\right) \quad \text { with } \quad V_{m, n} \stackrel{\text { def }}{=} g_{m}^{\text {true }} \mathcal{A}_{m, n}+b_{m}^{\text {true }} .
$$

The two main advantages of expressing the acceleration relative to the Earth gravity in (1) are that it simplifies the notation throughout this paper and, for SI units, $b_{m}$ and $g_{m}$ get the same units (V) as can be obtained from the data sheets of our acceleometers.

We address three main topics in this paper:

1. In Section 2.1, we derive simple conditions on the available measurements $V$, for which we provide simple formulas for unique calibration parameters $b_{m}$ and $g_{m}$ such that

$$
\left(\frac{V_{1, n}-b_{1}}{g_{1}}\right)^{2}+\left(\frac{V_{2, n}-b_{2}}{g_{2}}\right)^{2}+\left(\frac{V_{3, n}-b_{3}}{g_{3}}\right)^{2}=1, \quad n=1,2, \ldots, 6 .
$$

From the uniqueness and (1), it then clearly follows that $b_{m}=b_{m}^{\text {true }}$ and $g_{m}=g_{m}^{\text {true }}$.

\footnotetext{
${ }^{1}$ Percentages for the LIS3L02AQ accelerometer described at http://www.sunspotworld.com/docs/AppNotes/AccelerometerAppNote.pdf
} 
2. We show in Section 2.4 that there are nontrivial orientations of the accelerometer for which the measurements $V$ does not satisfy the conditions necessary to compute unique $b_{m}$ and $g_{m}$ satisfying (2). On the other hand, we also show that we always get unique $b_{m}$ and $g_{m}$ satisfying (2) for all "reasonably small" deviations from a natural measurement setup with 90 degree rotations of the accelerometer and one measurement axis aligned with the Earth gravity field. Here, "reasonably small" means, for example, at most $5^{\circ}$ deviation for the Colibrys SF3000L accelerometers in our lab.

3. In Section 2.3 we present Monte Carlo simulation results on the sensitivity of our basic algorithm and two least squares extensions of it to the measurement orientations, noise and cross-axis interference. In Section 2.5, a direct comparison of our explicit non-iterative solution with the iterative method proposed in [6] shows that the latter always converges in at most two iterations.

\section{Main results}

\subsection{The calibration method}

We will now derive a simple calibration method for triaxial accelerometers with perfectly orthogonal axes and voltage outputs satisfying the linear model (1c). It is natural and a simple task to set up the accelerometer and its wiring so that a large positive acceleration corresponds to a positive output voltage. We will therefore also assume the accelerometer to have positive gains.

The accelerometer voltage outputs $V$ in (1c) depend on $b_{m}^{\text {true }}, g_{m}^{\text {true }}$ and $\mathcal{A}_{m, n}$, all of which are unknown, but the following theorem shows that if $V$ satisfies a certain easily checked condition, then (6) gives $b_{m}=b_{m}^{\text {true }}$ and $g_{m}=g_{m}^{\text {true }}$.

Theorem 1. For any $3 \times 6$-matrix $V$ with real-valued entries, define

$$
V^{+} \stackrel{\text { def }}{=}\left[P_{2}\left(V^{\mathrm{T}}\right) \quad V^{\mathrm{T}}\right] \quad \text { with elementwise squaring } \quad\left(P_{2}(M)\right)_{m, n} \stackrel{\text { def }}{=} M_{m, n}^{2}
$$

There is a unique $\boldsymbol{u}=\left[\begin{array}{llllll}u_{1} & u_{2} & u_{3} & w_{1} & w_{2} & w_{3}\end{array}\right]^{T}$ such that (with $\operatorname{sgn}(0) \stackrel{\text { def }}{=} 0$ and, for $x \neq 0$, $\operatorname{sgn}(x) \stackrel{\text { def }}{=} x /|x|)$

$$
V^{+} \boldsymbol{u}=\mathbf{1} \quad \text { and } \quad 0 \neq \operatorname{sgn}\left(u_{m}\right)=\operatorname{sgn}\left(1+\frac{w_{1}^{2}}{4 u_{1}}+\frac{w_{2}^{2}}{4 u_{2}}+\frac{w_{3}^{2}}{4 u_{3}}\right) \quad \text { for } m=1,2,3
$$

if and only if there are two unique vectors $\boldsymbol{b}, \boldsymbol{g} \in \mathbb{R}^{3}$ with entries $b_{m}, g_{m}$ such that

$$
\sum_{m=1}^{3} \frac{\left(V_{m, n}-b_{m}\right)^{2}}{g_{m}^{2}}=1, \quad g_{m}>0 \quad \text { and } \quad \sum_{m=1}^{3} \frac{b_{m}^{2}}{g_{m}^{2}} \neq 1 \quad \text { for } m=1,2,3 \text {. }
$$

When these equivalent statements hold, the unique $b_{m}$ and $g_{m}$ satisfying (5) are

$$
b_{m}=-\frac{w_{m}}{2 u_{m}} \quad \text { and } \quad g_{m}=\sqrt{\frac{1+\sum_{k=1}^{3} \frac{w_{k}^{2}}{4 u_{k}}}{u_{m}}} .
$$


For the proof of Theorem 1 see Appendix A.

As explained in Remark 10 p. 6. the condition $\sum_{m=1}^{3} \frac{b_{m}^{2}}{g_{m}^{2}} \neq 1$ in (5) holds with probability 1 for an arbitrarily chosen accelerometer. Note also that this condition is always true, for example, for accelerometers with $\boldsymbol{b}^{\text {true }}$ having length less than the smallest $g_{m}^{\text {true }}$.

Further, we show in Theorem 2 how to "get rid of" the condition $\operatorname{sgn}\left(u_{m}\right)=\operatorname{sgn}\left(1+\sum_{k=1}^{3} \frac{w_{k}^{2}}{4 u_{k}}\right)$ in (44), hence reducing Theorem 1 to basically saying that we can recover the parameters $b_{m}=b_{m}^{\text {true }}$ and $g_{m}=g_{m}^{\text {true }}$ from the measurements $V$ if and only if the matrix $V^{+}$in (3) is invertible. Finally, in Section 2.4 we show one way to choose accelerometer calibration measurement orientations that guarantee the invertibility of $V^{+}$.

The following corollary of Theorem 1 will be useful in comparison of our results with the results obtained in [6].

Corollary 1. For arbitrary real-valued $3 \times 6$ matrices $V$ and $\Lambda$ such that $\Lambda_{m, n}=\frac{V_{m, n}-b_{m}^{(0)}}{g_{m}^{(0)}}$, define $V^{+} \stackrel{\text { def }}{=}\left[P_{2}\left(\Lambda^{T}\right) \Lambda^{T}\right]$ as in (3). Suppose that $b_{m}^{(0)}$ and $g_{m}^{(0)}$ are such that (4) holds for a unique $\boldsymbol{u}=\left[\begin{array}{llllll}u_{1} & u_{2} & u_{3} & w_{1} & w_{2} & w_{3}\end{array}\right]^{T}$. Then, $\sum_{m=1}^{3} \frac{\left(V_{m, n}-b_{m}\right)^{2}}{g_{m}^{2}}=1$ for

$$
b_{m} \stackrel{\text { def }}{=} b_{m}^{(0)}-\frac{w_{m}}{2 u_{m}} g_{m}^{(0)} \quad \text { and } \quad g_{m} \stackrel{\text { def }}{=} g_{m}^{(0)} \sqrt{\frac{1+\sum_{k=1}^{3} \frac{w_{k}^{2}}{4 u_{k}}}{u_{m}}} .
$$

Proof of Corollary [1. We know from (66) that for $b_{m}^{\prime}=-\frac{w_{m}}{2 u_{m}}$ and $g_{m}^{\prime}=\sqrt{\frac{1+\sum_{k=1}^{3} \frac{w_{k}^{2}}{4 u_{k}}}{u_{m}}}$,

$$
1=\sum_{m=1}^{3} \frac{\left(\Lambda_{m, n}-b_{m}^{\prime}\right)^{2}}{g_{m}^{\prime 2}}=\sum_{m=1}^{3} \frac{\left(\frac{V_{m, n}-b_{m}^{(0)}}{g_{m}^{(0)}}-b_{m}^{\prime}\right)^{2}}{g_{m}^{\prime 2}}
$$

from which (7) follows.

Now recall that Theorem 1 is supposed to be applied to real measurement data from an accelerometer that can be well modeled as having orthogonal axes and voltages given by the linear model (1c). Hence, we typically know from accelerometer data sheets and/or previous calibrations that there exists at least one choice of the parameters $b_{m}$ and $g_{m}$ (namely $b_{m}=b_{m}^{\text {true }}$ and $\left.g_{m}=g_{m}^{\text {true }}\right)$, for which $\sum_{m=1}^{3}\left(\frac{V_{m, n}-b_{m}}{g_{m}}\right)^{2}=1$ and $\sum_{k=1}^{3} \frac{b_{k}^{2}}{g_{k}^{2}} \neq 1$. By using this extra knowledge, we can basically remove the condition $0 \neq \operatorname{sgn}\left(u_{m}\right)=\operatorname{sgn}\left(1+\frac{w_{1}^{2}}{4 u_{1}}+\frac{w_{2}^{2}}{4 u_{2}}+\frac{w_{3}^{2}}{4 u_{3}}\right)$ from (4), so that unique $b_{m}$ and $g_{m}$ can be computed if and only if $V^{+}$is invertible. More precisely, we get the main theorem of this paper:

Theorem 2 (Calibration method). For any $3 \times 6$-matrix $V$ with real-valued elements, define the matrix

$$
V^{+} \stackrel{\text { def }}{=}\left[P_{2}\left(V^{\mathrm{T}}\right) \quad V^{\mathrm{T}}\right] \text { with elementwise squaring }\left(P_{2}(M)\right)_{m, n} \stackrel{\text { def }}{=} M_{m, n}^{2} .
$$

Assume that there exist vectors $\boldsymbol{b}, \boldsymbol{g} \in \mathbb{R}^{3}$ such that

$$
\sum_{m=1}^{3}\left(\frac{V_{m, n}-b_{m}}{g_{m}}\right)^{2}=1 \quad \text { and } \quad \sum_{k=1}^{3} \frac{b_{k}^{2}}{g_{k}^{2}} \neq 1 .
$$

Then,

$$
V^{+} \boldsymbol{u}=\mathbf{1} \text { has a unique solution } \boldsymbol{u}=\left[\begin{array}{llllll}
u_{1} & u_{2} & u_{3} & w_{1} & w_{2} & w_{3}
\end{array}\right]^{T}
$$


if and only if the vectors $\boldsymbol{b}, \boldsymbol{g} \in \mathbb{R}^{3}$ in (8) are unique.

When these equivalent statements hold, (6) gives the unique $b_{m}$ and $g_{m}$ satisfying (8).

For the proof of Theorem 2 see Appendix B. This proof also includes explicit formulas (34) for the inverse of the mapping $\boldsymbol{u} \rightarrow(\boldsymbol{b}, \boldsymbol{g})$ in (6) .

Note that in absence of noise and assuming the six parameter model (1c), equation (6) gives a simple formula that completely solves the calibration problem. In Sections 2.2 and 2.3. we reduce noise by computing time averages. One further way to extend the stability against additive independent noise is to do more than six measurements and then do a least squares solution of the equation $V^{+} \boldsymbol{u}=\mathbf{1}$ in (44) and (9).

Thus we arrive at the following simple calibration algorithm:

Step 1: Do $N \geq 6$ measurements with the accelerometer placed at rest in different orientations.

Step 2: Compute the mean value in $N$ intervals where the accelerometer can be considered to be at rest (as demonstrated in next subsection) and form the $3 \times N$ matrix $V$ from these voltages and the $N \times 6$-matrix $V^{+}$as defined in (3).

Step 3: Solve $V^{+} \boldsymbol{u}=\mathbf{1}$, for example, in the least-squares sense.

Step 4: Use (6) to compute $b_{m}$ and $g_{m}$.

The simulations in Section 2.3 include a comparison of this algorithm with an alternative least squares calibration approach that instead chooses $b_{m}, g_{m}$ for minimization of the error $\sum_{n=1}^{N}\left|1-\sum_{m=1}^{3}\left(\frac{V_{m, n}-b_{m}}{g_{m}}\right)^{2}\right|^{2}$ for $N \geq 6$, as was done for accelerometers with nonorthogonal axes in [8. More precisely, for $\Omega \stackrel{\text { def }}{=} 1-\sum_{m=1}^{3} \frac{b_{m}^{2}}{g_{m}^{2}}$ and $\boldsymbol{u} \stackrel{\text { def }}{=}\left[\frac{1}{g_{1}^{2} \Omega} \frac{1}{g_{2}^{2} \Omega} \frac{1}{g_{3}^{2} \Omega} \frac{-2 b_{1}}{g_{1}^{2} \Omega} \frac{-2 b_{2}}{g_{2}^{2} \Omega} \frac{-2 b_{3}}{g_{3}^{2} \Omega}\right]$ (cf. equation (34)), the aim then is to minimize

$$
\begin{aligned}
\sum_{n=1}^{N}\left(1-\sum_{m=1}^{3}\left(\frac{V_{m, n}-b_{m}}{g_{m}}\right)^{2}\right)^{2} & =\sum_{n=1}^{N}\left(1-\sum_{m=1}^{3} \frac{b_{m}^{2}}{g_{m}^{2}}-\sum_{m=1}^{3}\left(V_{m, n}^{2} \frac{1}{g_{m}^{2}}+V_{m, n} \cdot \frac{-2 b_{m}}{g_{m}^{2}}\right)\right)^{2} \\
& =\Omega^{2} \sum_{n=1}^{N}\left(1-\sum_{m=1}^{3}\left(V_{m, n}^{2} \frac{1}{g_{m}^{2} \Omega}+V_{m, n} \cdot \frac{-2 b_{m}}{g_{m}^{2} \Omega}\right)\right)^{2} \\
& =\Omega^{2}\left\|\mathbf{1}-V^{+} \boldsymbol{u}\right\|_{2}^{2}, \quad\left\|\left[x_{1} \cdots x_{N}\right]^{\mathrm{T}}\right\|_{2} \stackrel{\text { def }}{=} \sqrt{x_{1}^{2}+\cdots+x_{N}^{2}} .
\end{aligned}
$$

Hence, instead of choosing $b_{m}, g_{m}$ and thus also $\boldsymbol{u}$ for minimization of $\left\|\mathbf{1}-V^{+} \boldsymbol{u}\right\|_{2}^{2}$ in Step 3 of our algorithm, this alternative approach minimizes $\Omega^{2}\left\|\mathbf{1}-V^{+} \boldsymbol{u}\right\|_{2}^{2}$. We do this with the following simple and iterative but fast converging extension of Step 3:

Step 3a: Set $\Omega=1$.

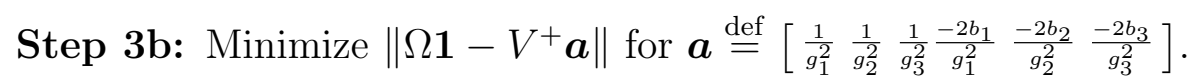

Step 3c: Compute $\Omega_{\mathrm{New}} \stackrel{\text { def }}{=} 1-\sum_{m=1}^{3} \frac{b_{m}^{2}}{g_{m}^{2}}$ from $\boldsymbol{a}$. If $\left|\Omega_{\mathrm{New}}-\Omega\right|>\epsilon$, then set $\Omega=\Omega_{\mathrm{New}}$ and go back to Step 3b. (The threshold $\epsilon=2.2 \cdot 10^{-13}$ in Section 2.3.)

We will from now on refer to this as the iterative least squares approach. 
Remark 1. The condition $\sum_{m=1}^{3} \frac{b_{m}^{2}}{g_{m}^{2}} \neq 1$ in (5) means that $\left(b_{1}, b_{2}, b_{3}\right)$ is a point not located on the ellipsoid surface $E_{\boldsymbol{g}} \stackrel{\text { def }}{=}\left\{\left(b_{1}, b_{2}, b_{3}\right) \in \mathbb{R}^{3} \mid \sum_{m=1}^{3} b_{m}^{2} / g_{m}^{2}=1\right\}$. This will always be true for any accelerometer with biases $b_{m}^{\text {true }}$ that are small enough compared to the gains, for example, in the sense that $\boldsymbol{b}^{\text {true }}$ has length $\sqrt{b_{1}^{\text {true }^{2}}+b_{2}^{\text {true }}{ }^{2}+b_{3}^{\text {true }} 2}<\min _{m} g_{m}^{\text {true }}$, or in the sense that each $\left|b_{m}\right| \leq g_{m} / 2$, as in Theorem 3, below. Both these conditions hold for the accelerometer calibrated in Section 2.2, as well as for the Monte Carlo simulations in Section 2.3.

Moreover, for a randomly chosen accelerometer, it should be safe to assume that the probability for the accelerometer to have bias $\boldsymbol{b}$ and gain $\boldsymbol{g}$ is described by an unknown but integrable probability density function $f(\boldsymbol{b}, \boldsymbol{g})$. Then, since the ellipsoid surface $E_{\boldsymbol{g}}$ has Lebesgue measure 0 in $\mathbb{R}^{3}$, , the probability for a randomly chosen accelerometer not to satisfy the condition $\sum_{m=1}^{3} \frac{b_{m}^{2}}{g_{m}^{2}} \neq 1$ in (5) is $\int_{\mathbb{R}_{+}^{3}} \int_{E_{\boldsymbol{g}}} f(\boldsymbol{b}, \boldsymbol{g}) d \boldsymbol{b} d \boldsymbol{g}=\int_{\mathbb{R}_{+}^{3}} 0 d \boldsymbol{g}=0$.

Remark 2. If some additional information on the gain factors is presented, then it is possible to reduce the number of measurements in order to compute the true bias and gain parameters in the following way. For example, if it is known in advance that all three gain factors are equal, then it is enough to make four measurements in order to find the unknown parameters. In this case Theorems 1 and 2 can be applied to the matrix $V^{+}$defined by

$$
V^{+}=\left(\begin{array}{llll}
V_{1,1}^{2}+V_{2,1}^{2}+V_{3,1}^{2} & V_{1,1} & V_{2,1} & V_{3,1} \\
V_{1,2}^{2}+V_{2,2}^{2}+V_{3,2}^{2} & V_{1,2} & V_{2,2} & V_{3,2} \\
V_{1,3}^{2}+V_{2,3}^{2}+V_{3,3}^{2} & V_{1,3} & V_{2,3} & V_{3,3} \\
V_{1,4}^{2}+V_{2,4}^{2}+V_{3,4}^{2} & V_{1,4} & V_{2,4} & V_{3,4}
\end{array}\right)
$$

and $\boldsymbol{u}=\left[\begin{array}{llll}u & w_{1} & w_{2} & w_{3}\end{array}\right]$. Thus,

$$
b_{m}=-\frac{w_{m}}{2 u}, \quad g=\sqrt{\frac{1+\sum_{k=1}^{3} \frac{w_{k}^{2}}{4 u}}{u}} .
$$

If instead, it is known that two of three gain factors are equal, then it is enough with five measurements to define the unknown parameters. For example, if the gain factors for the accelerometer in the $x$ - and $y$-directions are the same, then the following matrix can be considered

$$
V^{+}=\left(\begin{array}{ccccc}
V_{1,1}^{2}+V_{2,1}^{2} & V_{3,1}^{2} & V_{1,1} & V_{2,1} & V_{3,1} \\
V_{1,2}^{2}+V_{2,2}^{2} & V_{3,2}^{2} & V_{1,2} & V_{2,2} & V_{3,2} \\
V_{1,3}^{2}+V_{2,3}^{2} & V_{3,3}^{2} & V_{1,3} & V_{2,3} & V_{3,3} \\
V_{1,4}^{2}+V_{2,4}^{2} & V_{3,4}^{2} & V_{1,4} & V_{2,4} & V_{3,4} \\
V_{1,5}^{2}+V_{2,5}^{2} & V_{3,5}^{2} & V_{1,5} & V_{2,5} & V_{3,5}
\end{array}\right)
$$

and $\boldsymbol{u}=\left[\begin{array}{lllll}u_{1} & u_{3} & w_{1} & w_{2} & w_{3}\end{array}\right]$. Thus, assigning $u_{2}=u_{1}$

$$
b_{m}=-\frac{w_{m}}{2 u_{m}}, \quad g_{m}=\sqrt{\frac{1+\sum_{k=1}^{3} \frac{w_{k}^{2}}{4 u_{k}}}{u_{m}}} .
$$

Note also in these two cases that if no reduction in measurements is required, then Theorems 1 and 2 produce the correct result directly by considering six measurements.

\section{$2.2 \quad$ Experimental results}

We demonstrate our calibration method on a triaxial Colibrys SF3000L accelerometer, which was placed at rest in room temperature on each of its different sides for at least 30 seconds 


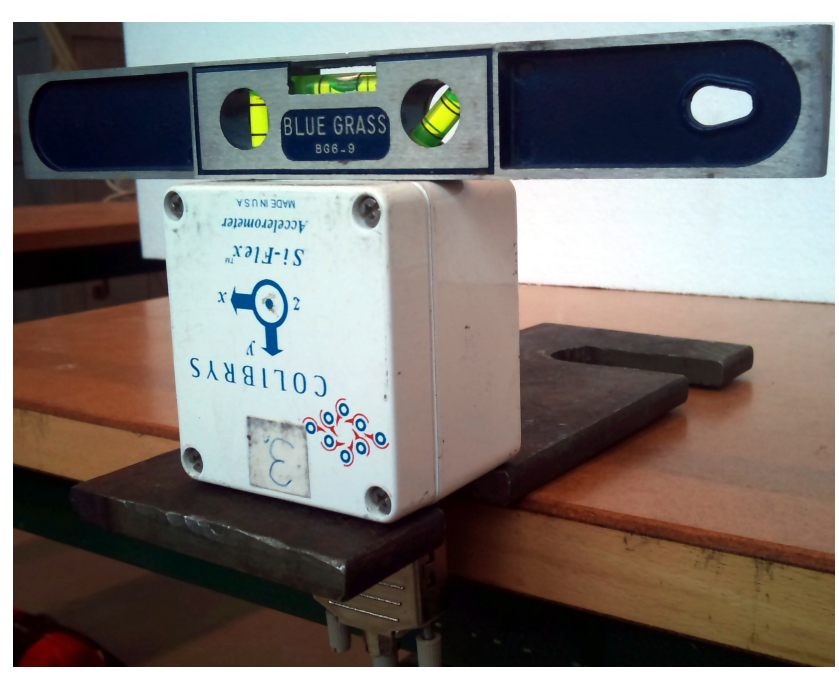

(a)

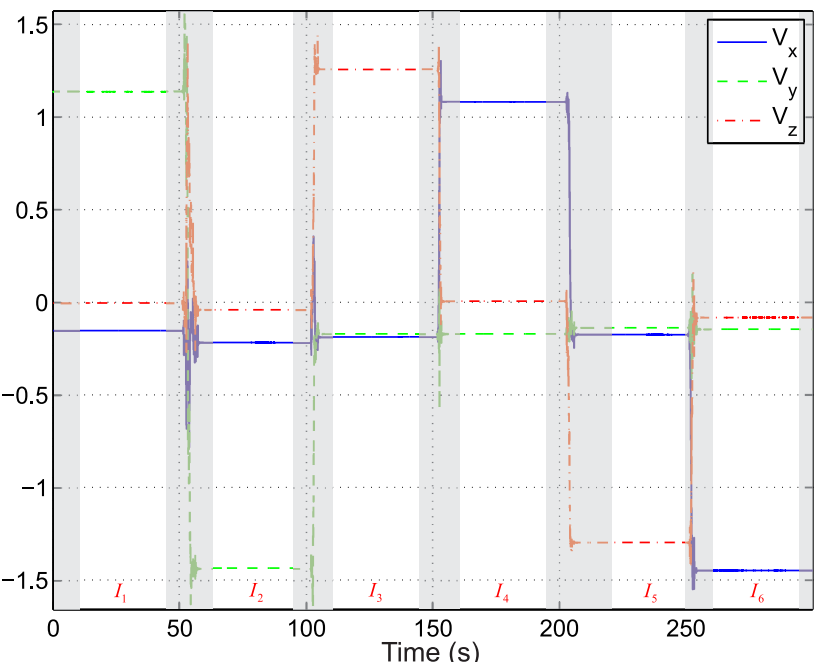

(b)

Figure 1: The measurements were done with one accelerometer placed on each of its six different sides on a table, resting at least 30 seconds on each side. (a) Measurement setup for the side with connecting cables. (b) Recorded voltage data.

of measuring. For the side with connecting cables, we used the simple holder in Figure 1 (a). No careful alignments with the horizontal plane are needed, since it is shown in Theorem 3 and demonstrated in Section 2.3 that horizontal alignment is good, but roughly horizontal is enough. Figure 1(b) shows the measured voltages. We ignore the greyed out sections where the accelerometer is rotated and consider the accelerometer to be at rest in the remaining intervals $I_{n}$. We compute the average voltage in each such interval to reduce the noise and thus get higher precision measurements. Then, Theorem 2 gives the gains and biases

$$
b_{1}=-0.1838, b_{2}=-0.1495, b_{3}=-0.0201, g_{1}=1.2662, g_{2}=1.2870 \text { and } g_{3}=1.2776 \text {. }
$$

In several independent calibrations with the same accelerometer, we got the same first two decimals for all $b_{m}$ and $g_{m}$. Hence we expect to have errors at least in the third decimal due to noise. The simulation results in next subsection gives further insight on the size of those errors.

\subsection{Monte Carlo simulation}

To further investigate how sensitive the different methods proposed in Section 2.1 are to noise and cross-axis interference, we have done Monte Carlo simulations based on the following model. For an accelerometer that is placed at rest in $N \geq 6$ different orientations, the measured dimensionless accelerations $\mathcal{A}_{m, n}$ of (1a) are stored as column vectors of a $3 \times N$ matrix $A$. Gain, bias and additive noise $v_{m, n}$ with variance $\sigma^{2}$ gives the voltages

$$
v_{m, n}[l]=\mathcal{A}_{m, n} g_{m}+b_{m}+\nu_{m, n}[l], \quad l=1,2,3, \ldots, L_{n},
$$

where $L_{n}$ is the number of samples collected for the $n$th orientation, or in other words, the length of the interval $I_{n}$ in Figure 1 (b). From these samples, we compute

$$
V_{m, n}^{\prime}=\frac{1}{L_{n}} \sum_{l=1}^{L_{n}} v_{m, n}[l] .
$$




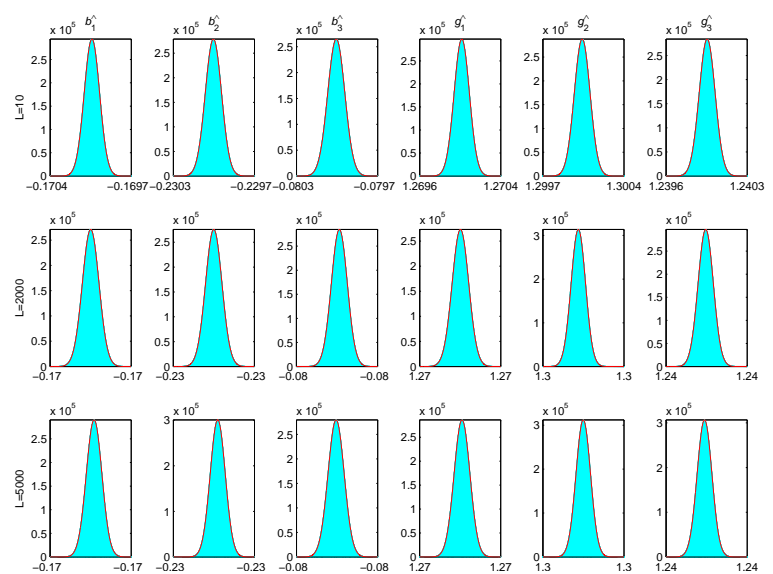

(a)

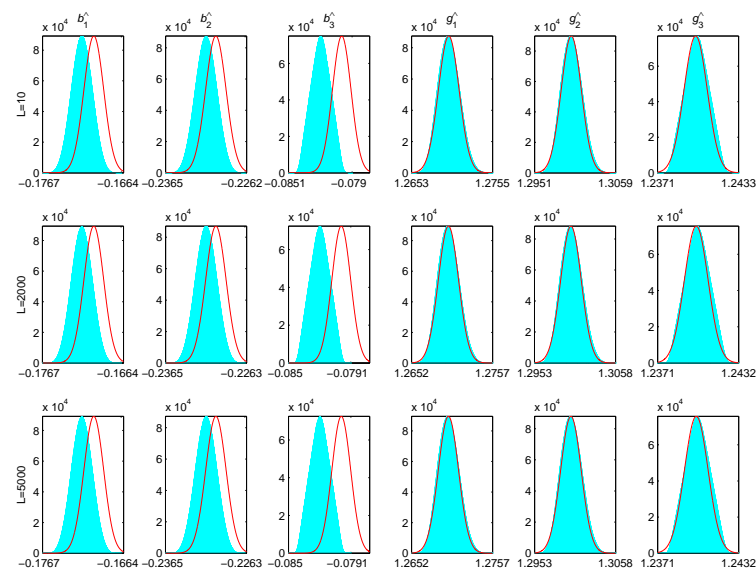

(b)

Figure 2: Histograms for the estimates $\widehat{b_{m}}$ and $\widehat{g_{m}}$ (shaded) show a very good correspondence with Gaussian distributions (solid lines). (a) For noise standard deviation $\sigma=0.0003$ and no cross-axis interference. (b) For noise standard deviation $\sigma=0.0003$ and cross-axis interference $0.5 \%$.

For some nonnegative constant $C$, we model the cross-axis interference as the following leakage between the axes:

$$
V_{m, n}=V_{m, n}^{\prime}+C \sum_{1 \leq d \leq 3, d \neq m} V_{d, n}^{\prime}, \quad m=1,2,3, \quad n=1,2, \ldots, N, \quad C \geq 0 .
$$

For our accelerometers and laboratory measurements, $C<10^{-2.3} \approx 0.5 \%$ and $\sigma \approx 0.0003 \mathrm{~V}$, but we will also compare how the investigated calibration methods perform in a more noisy environment, as well as for $C=0$. We model the estimates $\widehat{b_{m}}$ and $\widehat{g_{m}}$ of $b_{m}$ and $g_{m}$ as samples from independent stationary ergodic processes $B_{m, k}$ and $G_{m, k}$, respectively, with expectation $\mathrm{E}\left[B_{m, k}\right]=\lim _{K \rightarrow \infty} \frac{1}{K} \sum_{k=1}^{K} B_{m, k}$ and variance $\operatorname{Var}\left(B_{m, k}\right)=\lim _{K \rightarrow \infty} \frac{1}{K-1} \sum_{k=1}^{K}\left|B_{m, k}-\mathrm{E}\left[B_{m, k}\right]\right|^{2}$.

\section{Number of Monte Carlo iterations needed for convergence}

In Figure 3, some error expectations $\left|\mathrm{E}\left[B_{m, k}-b_{m}\right]\right|$ are about $10^{3}$ times smaller than the corresponding standard deviations $\sqrt{\operatorname{Var}\left(B_{m, k}-b_{m}\right)}=\sqrt{\operatorname{Var}\left(B_{m, k}\right)}$. Thus, for the computed and plotted estimate $\frac{1}{K} \sum_{k=1}^{K}\left(B_{m, k}-b_{m}\right) \approx \mathrm{E}\left[B_{m, k}-b_{m}\right]$ to have sufficient accuracy, one can decide to choose $K$ so that the standard deviation of $\frac{1}{K} \sum_{k=1}^{K}\left(B_{m, k}-b_{m}\right)$ is, say, $10^{4}$ times smaller than the standard deviation $\sqrt{\operatorname{Var}\left(B_{m, k}\right)}$ (corresponding a correct first digit), that is, that $K$ is chosen so that

$$
10^{-8} \operatorname{Var}\left(B_{m, k}\right) \geq \operatorname{Var}\left(\frac{1}{K} \sum_{k=1}^{K} B_{m, k}\right)=\frac{K \operatorname{Var}\left(B_{m, k}\right)}{K^{2}}=\frac{1}{K} \operatorname{Var}\left(B_{m, k}\right) .
$$

This suggests a need for roughly $K=10^{8}$ Monte Carlo iterations in Figure 3 ,

\section{Accelerometer orientations}

We will compare some different choices of accelerometer orientations that we for brevity will refer to as 90 degree rotations and random orientations. 
With 90 degree rotations we mean the ideal recommended six orientations with the accelerometer resting on one perfectly horizontal side in each measurement.

$N$ random orientations denotes a more realistic approach with the accelerometer resting on its six sides for the first six measurement but with a deviation from the horizontal plane that is uniformly distributed in the range of \pm 10 degrees in spherical coordinates $\left[\begin{array}{lll}x & y & z\end{array}\right]=$ $[\cos (\beta) \cos (\alpha) \cos (\beta) \sin (\alpha) \sin (\beta)]$. Orientations 7 to $N$ are random and uniformly distributed on the unit sphere.

We have also investigated an alternative approach with $N$ orientations uniformly distributed on the unit sphere, but this gave highly unstable calibration results. This can be an expected consequence of ill-conditioned matrices $V^{+}$for orientations close to those generating singular $V^{+}$, such as the one in the beginning of Section 2.4.
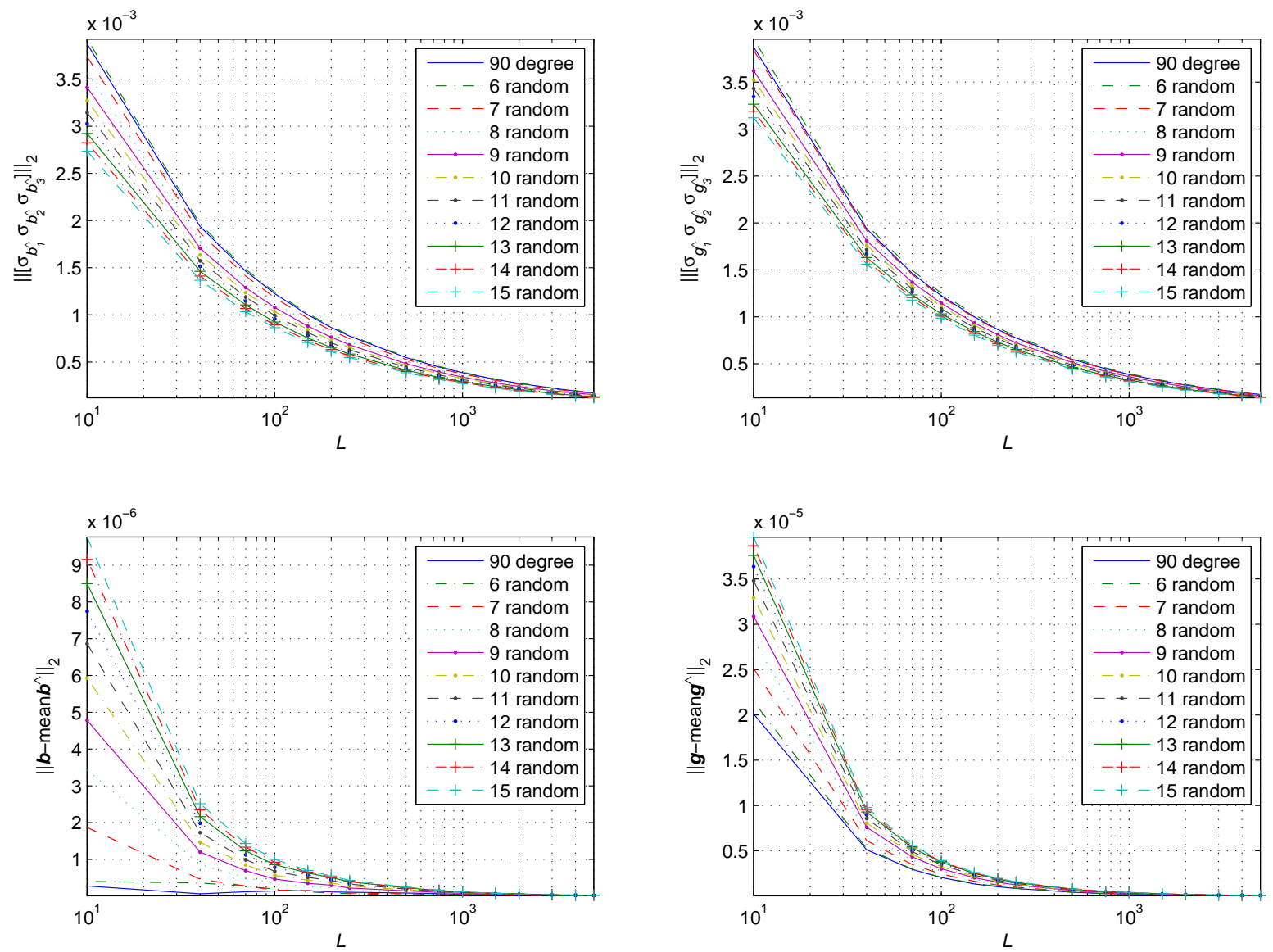

Figure 3: Standard deviation and expectation of the error of the estimates $\widehat{b_{m}}$ and $\widehat{g_{m}}$. Noniterative least squares, $\sigma=0.01$, no cross-axis interference and 127300000 Monte Carlo iterations.

\section{Simulation results}

As illustrated for two typical examples in Figure 2, the estimates $\widehat{b_{m}}$ and $\widehat{g_{m}}$ coincide well with Gaussian probability distributions with very small estimation biases in absence of crossaxis interference. Hence the size of the estimation error is almost completely determined by this standard deviation in these cases, whereas with the cross-axis interference present, the estimation bias is of the same order of magnitude, so that both together decide the size of the estimation error. 

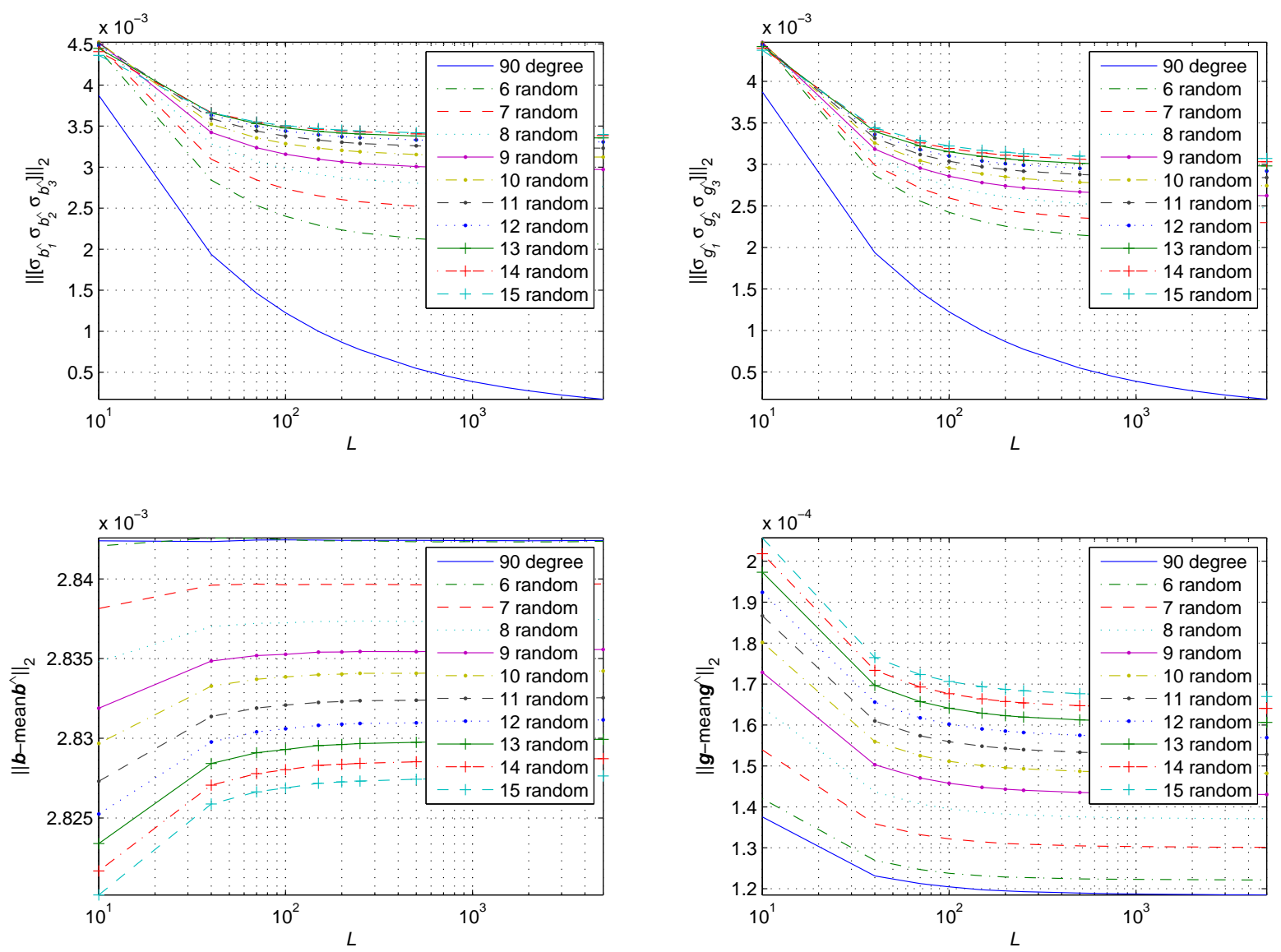

Figure 4: Standard deviation and expectation of the errors of the estimates $\widehat{b_{m}}$ and $\widehat{g_{m}}$. Noniterative least squares, $\sigma=0.01,0.5 \%$ cross-axis interference, and 44750000 Monte Carlo iterations.

For investigating the sensitivity of the methods to noise and cross-axis interference, we have performed large scale Monte Carlo simulations for constant $L_{n}=L$ in (13) and accelerometer gains and biases

$$
\boldsymbol{b}^{\text {true }}=\left[\begin{array}{lll}
-0.17 & -0.23 & -0.08
\end{array}\right]^{\mathrm{T}} \quad \text { and } \quad \boldsymbol{g}^{\text {true }}=\left[\begin{array}{lll}
1.27 & 1.30 & 1.24
\end{array}\right]^{\mathrm{T}},
$$

similar to one of the accelerometers in our lab. This leads to the following main comparisons and conclusions:

No cross-axis interference. For noise standard deviation $\sigma=0.01$, the topmost plots in Figure 3 , show how the standard deviation of $\widehat{b_{m}}$ and $\widehat{g_{m}}$ decay with $L$. The lowermost plots show how the estimation biases $\mathrm{E}\left[\widehat{b_{m}}-b_{m}^{\text {true }}\right]$ and $\mathrm{E}\left[\widehat{g_{m}}-g_{m}^{\text {true }}\right]$ decay with $L$. The plots for $x$-, $y$ - and $z$-direction look the same and are all incorporated here via the $l^{2}$ norm $\left\|\left[\begin{array}{lll}a_{x} & a_{y} & a_{z}\end{array}\right]\right\|_{2} \stackrel{\text { def }}{=} \sqrt{a_{x}^{2}+a_{y}^{2}+a_{z}^{2}}$. The estimation error is completely dominated by the standard deviation and the plotted non-iterative least squares approach shows some clear but not very large improvement, as compared to six measurements and 90 degree rotations.

Adding cross-axis interference: For the same $\sigma$ but with $0.5 \%$ cross-axis interference added, Figure 4 shows that all methods now give much larger estimation bias $\mathrm{E}\left[\widehat{b_{m}}-b_{m}^{\text {true }}\right]$ 

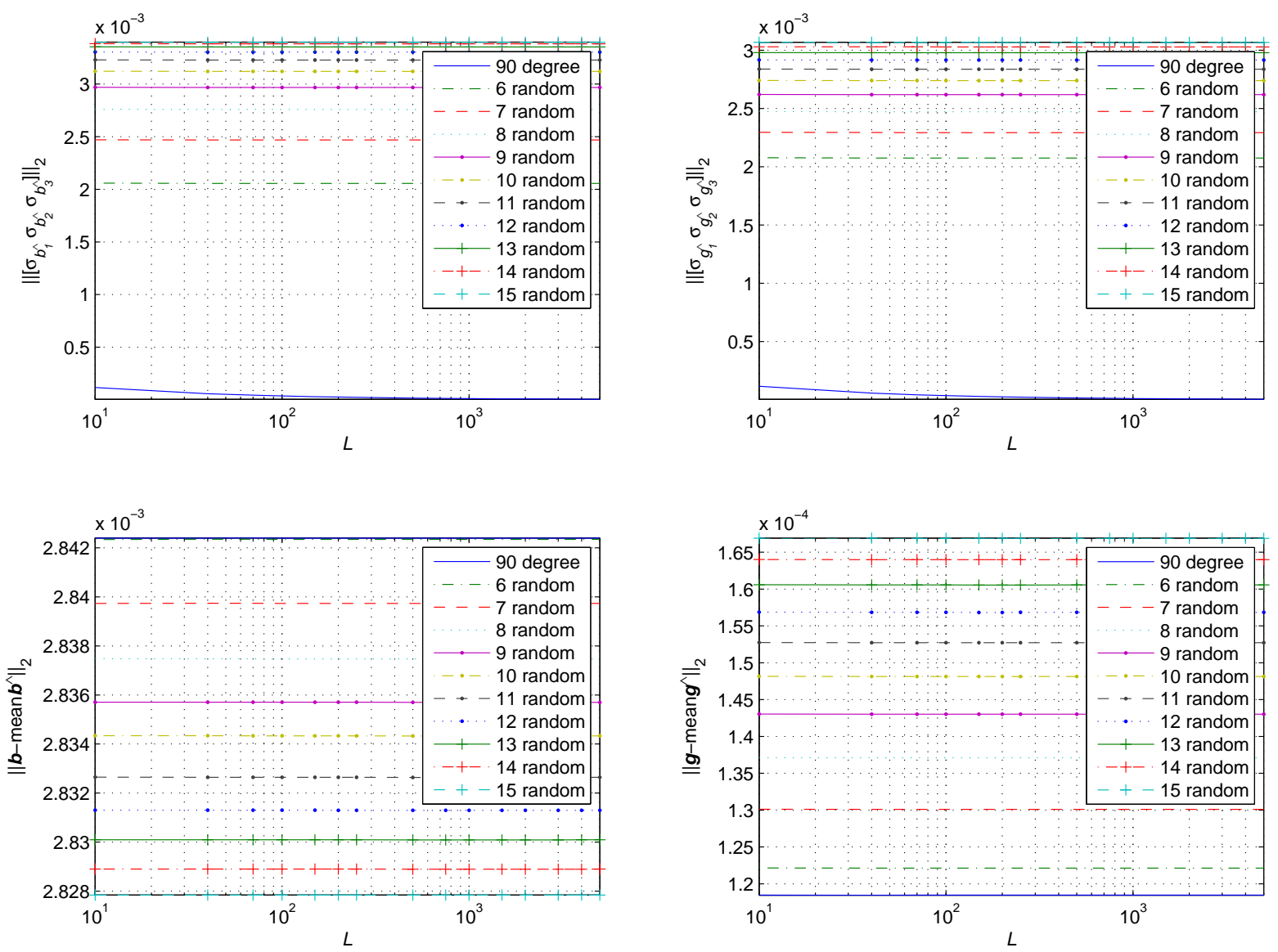

Figure 5: Standard deviation and expectation of the errors of the estimates $\widehat{b_{m}}$ and $\widehat{g_{m}}$. Noniterative least squares, $\sigma=0.0003,0.5 \%$ cross-axis interference, and 43425000 Monte Carlo iterations.

and $\mathrm{E}\left[\widehat{g_{m}}-g_{m}^{\text {true }}\right]$, affecting the estimation error almost as much as the plotted standard deviations, but the approach with 90 degree rotations now is clearly the best one. In fact, this method is largely unaffected by the cross-axis interference.

A bit surprising for the least squares approaches is that the estimation biases $\mathrm{E}\left[\widehat{b_{m}}-b_{m}^{\text {true }}\right]$ are increasing functions of $L$, but at the same time with the standard deviation decreasing at a higher rate, so the total resulting estimation errors will still decrease with increasing $L$.

Non-iterative vs. iterative least squares: Exactly the same simulation for the iterative least squares calibration gives plots nearly identical to Figures 3 and 4 .

Lower noise levels: With $\sigma=0.0003$ as in the laboratory results in Section 2.2, and with no cross-axis interference, the errors are smaller but with similar behavior as in Figure 3 .

For cross-axis interference $0.5 \%$, Figure 5 shows, quite contrary to Figure 4 , that computing averages of more than $L=10$ samples only gives a very small additional reduction of the estimation error. The predicted estimation error is of the same size $10^{-3}$ that we observed in our experimental results in Section 2.2.

If we choose the largest $L$ in each of Figure 3, 4 and 5, sum the plotted estimation bias and standard deviation for each $\widehat{b_{m}}, \widehat{g_{m}}$ and each method, we get an estimated total estimation error, 


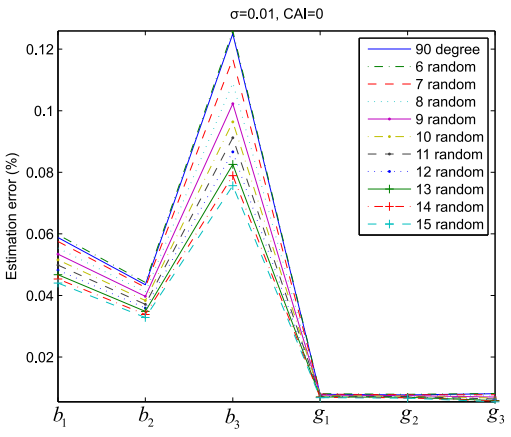

(a)

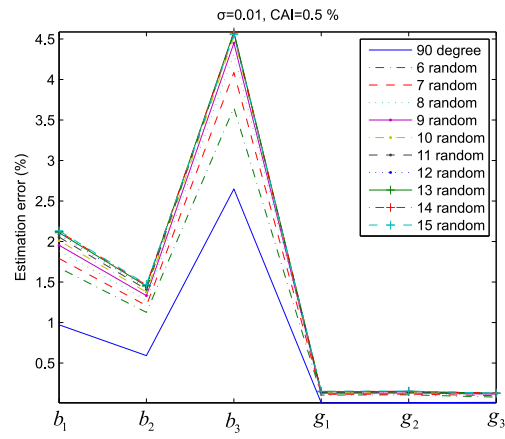

(b)

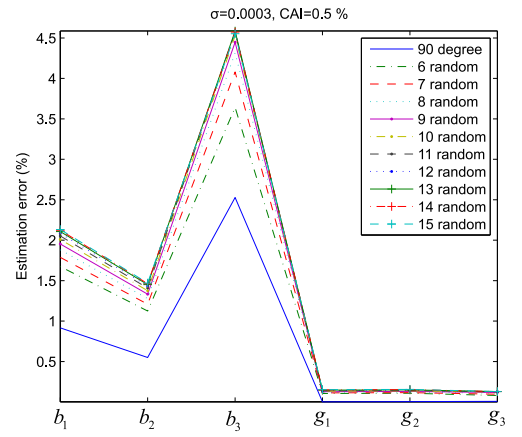

(c)

Figure 6: Estimated relative errors $\frac{\left|\mathrm{E}\left[\widehat{b_{m}}-b_{m}^{\text {true }}\right]\right|+\operatorname{Var}\left(\widehat{b_{m}}\right)^{1 / 2}}{b_{m}^{\text {true }}}$ and $\frac{\left|\mathrm{E}\left[\widehat{g_{m}}-g_{m}^{\text {true }}\right]\right|+\operatorname{Var}\left(\widehat{g_{m}}\right)^{1 / 2}}{g_{m}^{\text {true }}}$ plotted as percentages for all $L_{n}=5000$ in (13) and the same simulation setups as in (a) Figure 3. (b) Figure 4 and (c) Figure 5 .

which we can divide with $b_{m}^{\text {true }}$ and $g_{m}^{\text {true }}$ from (14) to get the relative errors that are plotted in Figure 6 .

These clearly indicate that all investigated methods work fine, that the least squares approach for more than six measurements gives better estimates when noise but no cross-axis interference is present, but in the more realistic scenario with cross-axis interference present, the idealized setup with six measurements and two horizontal axes perform better than the least squares approaches.

For example, Figure [ (b) shows that the relative error of the gain parameters are within $0.010-0.016 \%$ for calibration from 90 degrees rotations and six measurements with $0.5 \%$ crossaxis interference and noise standard deviation 0.01. The relative errors of the corresponding biases are up to $2.65 \%$ for the smallest bias $b_{3}^{\text {true }}=-0.008$ in (14), which is a much larger percentage, but since $b_{3}^{\text {true }}$ is so small, it still only gives a contribution of magnitude $10^{-4}=$ $10^{-4} \sqrt{\mathcal{A}_{1, n}^{2}+\mathcal{A}_{2, n}^{2}+\mathcal{A}_{3, n}^{2}}$ to the resulting error in the acceleration measurements $\mathcal{A}_{3, n}=\frac{V_{3, n}-b_{3}}{g_{3}}$. Thus this error is of the same order of magnitude as the corresponding error contribution $\max \left(\frac{1}{1.24}-\frac{1}{1.24+0.00016}, \frac{1}{1.24+0.00016}-\frac{1}{1.24}\right) \approx 1.04 \cdot 10^{-4}$ from $g_{3}$.

\subsection{Choosing accelerometer orientations for safe recovery of $b_{m}^{\text {true }}$ and $g_{m}^{\text {true }}$}

For successful application of Theorems 1 and 2, it is important to do the calibration measurements with the six orientations of the accelerometer chosen in such a way that the matrix $V^{+} \stackrel{\text { def }}{=}\left[P_{2}\left(V^{\mathrm{T}}\right) V^{\mathrm{T}}\right]$ is invertible. This is not the case, for example, if the measured accelerations are the column vectors of the matrix

$$
A=\left(\begin{array}{cccccc}
\frac{1}{\sqrt{2}} & \frac{1}{\sqrt{2}} & \frac{1}{\sqrt{2}} & -\frac{1}{\sqrt{2}} & -\frac{1}{\sqrt{2}} & -\frac{1}{\sqrt{2}} \\
\frac{1}{\sqrt{2}} & -\frac{1}{\sqrt{2}} & 0 & -\frac{1}{\sqrt{2}} & \frac{1}{\sqrt{2}} & 0 \\
0 & 0 & \frac{1}{\sqrt{2}} & 0 & 0 & -\frac{1}{\sqrt{2}}
\end{array}\right)
$$


For an accelerometer with gains $g_{m}^{\text {true }}=\sqrt{2}$ and biases $b_{m}^{\text {true }}=0$, the corresponding measured voltages would be

$$
V=\left(\begin{array}{cccccc}
1 & 1 & 1 & -1 & -1 & -1 \\
1 & -1 & 0 & -1 & 1 & 0 \\
0 & 0 & 1 & 0 & 0 & -1
\end{array}\right)
$$

It is easy to check that $V^{+}$has linearly dependent rows $\left(\right.$row $_{1}=\operatorname{row}_{2}-$ row $_{4}+$ row $\left._{5}\right)$. Hence, by Theorem 2 there is no unique choice of gains and biases giving an acceleration matrix $\left(A_{\boldsymbol{b}, \boldsymbol{g}}\right)_{m, n} \stackrel{\text { def }}{=} \frac{V_{m, n}-b_{m}}{g_{m}}$ with column vectors of length 1 . In this particular example, one choice is $g_{m}=g_{m}^{\text {true }}=\sqrt{2}$ and $b_{m}=b_{m}^{\text {true }}=0$, but the alternative choice $b_{m}=0, g_{1}=2 / \sqrt{3}$ and $g_{2}=g_{3}=2$ will also give an acceleration matrix with column vectors of length 1 .

A natural and simple calibration setup for a rectangular shaped accelerometer casing is to do the measurements with the accelerometer resting on each of its six different sides. The matrix $A$ in (15) is then "close" to the matrix $A_{0}=\left(I_{3 \times 3}-I_{3 \times 3}\right)$, or to another matrix with the same column vectors but in a different order. $A_{0}$ has the largest possible minimal angle $90^{\circ}$ between two column vectors, and for $b_{m}^{\text {true }}=0$ and $g_{m}^{\text {true }}=1$ it gives an orthogonal matrix $V^{+}$in Theorems 1 and 2. Thus, for $A$ close to $A_{0}$, corresponding to small variations of the rotations, gains and biases, we could expect some robustness in the sense that the invertibility of $V^{+}$is preserved. Indeed, for any $b_{m}^{\text {true }}$ and $g_{m}^{\text {true }}$ such that $\sum_{m=1}^{3} \frac{b_{m}^{\text {true }}{ }^{\text {true }}}{g_{m}^{\text {true }}} \neq 1$, Theorem 3 below guarantees $V^{+}$to be invertible for any $A$ with column vectors deviating from the corresponding column vector of $A_{0}$ with arbitrary angles less than a certain upper bound $\alpha$.

Thus a roughly horizontal alignment is enough and for any choice of orientations Theorem 2 immediately tells that unique $b_{m}$ and $g_{m}$ can be computed if and only if $V^{+}$is invertible. Recall from Section 2.3, however, that our simulation results indicate that nearly horizontal alignment gives improved robustness against cross-axis interference, and that a completely random choice of accelerometer orientations gives numerically highly unstable calibration results.

\section{Invertibility of the voltage matrix $V^{+}$in Theorem 2}

In the following theorem, we will consider the measured accelerations to be some approximation $A$ of the matrix $A_{0}=\left(\begin{array}{ll}I_{3 \times 3} & -I_{3 \times 3}\end{array}\right)$, that is, $A_{m, n}=A_{0 m, n}+\varepsilon_{m, n}$. For an accelerometer with bias and positive gain factors stored in the vectors $\boldsymbol{b}$ and $\boldsymbol{g}$, respectively, the measured voltages will be

$$
V_{A, \boldsymbol{b}, \boldsymbol{g}} \stackrel{\text { def }}{=}\left(\begin{array}{ccc}
g_{1} & 0 & 0 \\
0 & g_{2} & 0 \\
0 & 0 & g_{3}
\end{array}\right) A+\left(\begin{array}{llllll}
\boldsymbol{b} & \boldsymbol{b} & \boldsymbol{b} & \boldsymbol{b} & \boldsymbol{b} & \boldsymbol{b}
\end{array}\right) .
$$

Theorem 3 (Robust calibration measurement setups). For $m=1,2,3$ and $b_{m}, g_{m} \in \mathbb{R}$ such that $g_{m}>0$, suppose that $\frac{\left|b_{m}\right|}{g_{m}} \leq \beta \leq \frac{1}{2}$. Then, $S \stackrel{\text { def }}{=} \frac{b_{1}^{2}}{g_{1}^{2}}+\frac{b_{2}^{2}}{g_{2}^{2}}+\frac{b_{3}^{2}}{g_{3}^{2}}<1$ and $V_{A_{0}, \boldsymbol{b}, \boldsymbol{g}}^{+}$is invertible for $A_{0} \stackrel{\text { def }}{=}\left(I_{3 \times 3}-I_{3 \times 3}\right)$. Define

$$
\sigma \stackrel{\text { def }}{=} \min _{1 \leq m \leq 3} g_{m}, \quad \gamma \stackrel{\text { def }}{=} \max _{1 \leq m \leq 3} g_{m} \quad \text { and } \quad \Omega=\max \left\{1+3 \beta-3 \beta^{2}, \gamma\left(1+3 \beta^{2}\right)\right\} .
$$

For an arbitrary $3 \times 6$-matrix $A$, define

$$
\varepsilon_{m, n} \stackrel{\text { def }}{=}\left(A-A_{0}\right)_{m, n} \quad \text { and } \quad \varepsilon \stackrel{\text { def }}{=} \max _{1 \leq m \leq 3,1 \leq n \leq 6}\left|\varepsilon_{m, n}\right|
$$


If $\varepsilon$ satisfies

$$
0 \leq \varepsilon<\frac{1}{6}\left(-\left(6 \beta+2+\frac{3}{\gamma}\right)+\sqrt{\left(6 \beta+2+\frac{3}{\gamma}\right)^{2}+\frac{12 \sigma^{2}\left(1-3 \beta^{2}\right)}{\gamma^{2} \Omega}}\right),
$$

Then (17) defines an invertible matrix $V_{A, \boldsymbol{b}, \boldsymbol{g}}^{+}$.

Moreover, if the matrix A has column vectors of length 1, then a sufficient condition for (18) to hold is that the angle between each column vector in $A_{0}$ and the corresponding column vector in $A$ is at most $\alpha=2 \sin ^{-1}(\varepsilon / 2)$.

For the proof, see Appendix C. In particular, for the calibration results in (12), we get the bounds

$$
\beta>\frac{0.239}{1.230} \approx 0.20, \quad \gamma>1.29, \quad \sigma<1.20, \quad \Omega>1.48, \quad \varepsilon<0.0888, \quad \alpha<5.09^{\circ} .
$$

For the $8 \mathrm{~cm}$ casing side lengths of our Colibrys SF3000L accelerometers, $\pm 5.09^{\circ}$ misalignment corresponds to a $\pm 7.1 \mathrm{~mm}$ vertical movement of one side of the casing. This means that it is a relatively simple task to place the accelerometer "close enough" to being horizontal for Theorem 3 to guarantee that the gains and biases can be computed from the accelerometer voltage outputs.

\subsection{Comparison with a related iterative calibration method}

Our calibration method is closely related to a remarkably fast converging iterative calibration method proposed by Won and Golnaraghi in [6]. Their algorithm starts from initial guesses $\hat{b}_{m}^{(0)}$ and $\hat{g}_{m}^{(0)}$. At the $k$ th iteration, the estimated bias and gain is $\hat{b}_{m}^{(k)}$ and $\hat{g}_{m}^{(k)}$, which gives estimated accelerations $\hat{A}_{m, n}^{(k)} \stackrel{\text { def }}{=} \frac{V_{m, n}-\hat{b}_{m}^{(k)}}{\hat{g}_{m}^{(k)}}$.

A crucial idea in [6] is for each iteration to do the calibration parameter updating

$$
\hat{b}_{m}^{(k)}=\hat{b}_{m}^{(k-1)}+\tilde{b}_{m}^{(k)}, \quad \hat{g}_{m}^{(k)}=\hat{g}_{m}^{(k-1)} \tilde{g}_{m}^{(k)}
$$

and investigate how to choose the updating parameters $\tilde{g}_{m}^{(k)}$ and $\tilde{b}_{m}^{(k)}$ for making the algorithm to converge in the $k$ th step. This would mean that the $k$ th estimates $\hat{b}_{m}^{(k)}$ and $\hat{g}_{m}^{(k)}$ coincide with the true biases and gains, and consequently, the error after the $(k-1)$ th iteration is the vector $\boldsymbol{E}^{(k-1)}$ with $n$th entry

$$
\begin{aligned}
E_{n}^{(k-1)} & \stackrel{\text { def }}{=}\left(\hat{A}_{1, n}^{(k-1)}\right)^{2}+\left(\hat{A}_{2, n}^{(k-1)}\right)^{2}+\left(\hat{A}_{3, n}^{(k-1)}\right)^{2}-1 \\
& =\sum_{m=1}^{3}\left(\hat{A}_{m, n}^{(k-1)}\right)^{2}-\sum_{m=1}^{3}\left(\hat{A}_{m, n}^{(k)}\right)^{2}, \quad n=1, \ldots, 6 .
\end{aligned}
$$

Insertion of $\hat{A}_{m, n}^{(k)}=\frac{V_{m, n}-\hat{b}_{m}^{(k-1)}+\hat{b}_{m}^{(k-1)}-\hat{b}_{m}^{(k)}}{\hat{g}_{m}^{(k-1)} \hat{g}_{m}^{(k)} / \hat{g}_{m}^{(k-1)}}=\frac{\hat{A}_{m, n}^{(k-1)}}{\tilde{g}_{m}^{(k)}}-\frac{\tilde{b}_{m}^{(k)}}{\hat{g}_{m}^{(k-1)} \tilde{g}_{m}^{(k)}}$ in (22) gives

$$
E_{n}^{(k-1)}=\sum_{m=1}^{3}\left(1-\frac{1}{\left(\tilde{g}_{m}^{(k)}\right)^{2}}\right)\left(\hat{A}_{m, n}^{(k-1)}\right)^{2}+\frac{2 \tilde{b}_{m}^{(k)}}{\hat{g}_{m}^{(k-1)}\left(\tilde{g}_{m}^{(k)}\right)^{2}} \hat{A}_{m, n}^{(k-1)}-\frac{\left(\tilde{b}_{m}^{(k)}\right)^{2}}{\left(\hat{g}_{m}^{(k-1)} \tilde{g}_{m}^{(k)}\right)^{2}}
$$

The algorithm converges if each $\tilde{g}_{m}^{(k)}$ converges to 1 and each $\tilde{b}_{m}^{(k)}$ converges to 0 when $k \rightarrow \infty$. Then also the nonlinear last term in (23) will converge to 0 , and thus removing it gives a 
reasonable linearization of (23) to the equation

$$
\boldsymbol{E}^{(k-1)}=\Lambda^{(k-1)} \boldsymbol{c}^{(k)}
$$

with $\quad \Lambda^{(k)} \stackrel{\text { def }}{=}\left[P_{2}\left(\left(\hat{A}^{(k)}\right)^{T}\right)\left(\hat{A}^{(k)}\right)^{T}\right], \quad\left(P_{2}(M)\right)_{m, n} \stackrel{\text { def }}{=} M_{m, n}^{2}$,

and $\quad \boldsymbol{c}^{(k)} \stackrel{\text { def }}{=}\left(1-\frac{1}{\left(\tilde{g}_{1}^{(k)}\right)^{2}} 1-\frac{1}{\left(\tilde{g}_{2}^{(k)}\right)^{2}} 1-\frac{1}{\left(\tilde{g}_{3}^{(k)}\right)^{2}} \frac{2 \tilde{b}_{1}^{(k)}}{\hat{g}_{1}^{(k-1)}\left(\tilde{g}_{1}^{(k)}\right)^{2}} \frac{2 \tilde{b}_{2}^{(k)}}{\hat{g}_{2}^{(k-1)}\left(\tilde{g}_{2}^{(k)}\right)^{2}} \frac{2 \tilde{b}_{3}^{(k)}}{\hat{g}_{3}^{(k-1)}\left(\tilde{g}_{3}^{(k)}\right)^{2}}\right)^{\mathrm{T}}$.

The linear equation (24) is solved in each iteration of the algorithm proposed in 60. To simplify a direct comparison with Theorem 2 and Corollary 1 we observe from the definition (21) that $\boldsymbol{E}^{(k-1)}=\Lambda^{(k-1)}\left(\begin{array}{llllllll}1 & 1 & 1 & 0 & 0 & 0\end{array}\right)^{T}-\left(\begin{array}{llllll}1 & 1 & 1 & 1 & 1 & 1\end{array}\right)^{T}$, which on insertion in (24) gives

$$
\begin{aligned}
\Lambda^{(k-1)}\left(\begin{array}{llllll}
1 & 1 & 1 & 0 & 0 & 0
\end{array}\right)^{T}-\left(\begin{array}{llllll}
1 & 1 & 1 & 1 & 1 & 1
\end{array}\right)^{T} & =\Lambda^{(k-1)} \boldsymbol{c}^{(k)} \\
\Lambda^{(k-1)} \boldsymbol{u}^{(k)} & =\mathbf{1}
\end{aligned}
$$

with $\boldsymbol{u}^{(k)}=\left(\frac{1}{\left(\tilde{g}_{1}^{(k)}\right)^{2}} \frac{1}{\left(\tilde{g}_{2}^{(k)}\right)^{2}} \frac{1}{\left(\tilde{g}_{3}^{(k)}\right)^{2}}-\frac{2 \tilde{b}_{1}^{(k)}}{\hat{g}_{1}^{(k-1)}\left(\tilde{g}_{1}^{(k)}\right)^{2}}-\frac{2 \tilde{b}_{2}^{(k)}}{\hat{g}_{2}^{(k-1)}\left(\tilde{g}_{2}^{(k)}\right)^{2}}-\frac{2 \tilde{b}_{3}^{(k)}}{\hat{g}_{3}^{(k-1)}\left(\tilde{g}_{3}^{(k)}\right)^{2}}\right)^{T}$. We can thus rewrite (20) in terms of $\boldsymbol{u}^{(k)}$ :

$$
\begin{aligned}
& \hat{b}_{m}^{(k)}=\hat{b}_{m}^{(k-1)}-\frac{w_{m}^{(k)}}{2 u_{m}^{(k)}} \hat{g}_{m}^{(k-1)} \quad \text { and } \quad \hat{g}_{m}^{(k)}=\frac{\hat{g}_{m}^{(k-1)}}{\sqrt{u_{m}^{(k)}}} \\
& \text { with } \quad \boldsymbol{u}^{(k)} \stackrel{\text { def }}{=}\left(u_{1}^{(k)} u_{2}^{(k)} u_{3}^{(k)} w_{1}^{(k)} w_{2}^{(k)} w_{3}^{(k)}\right) .
\end{aligned}
$$

The resulting algorithm goes as follows

Choose real-valued initial values $\hat{b}_{m}^{(0)}$ and $\hat{g}_{m}^{(0)}>0$ such that $\Lambda^{(0)} \boldsymbol{u}^{(1)}=\mathbf{1}$ has a unique solution with $u_{m}^{(1)}>0$ for $m=1,2,3$.

The $k$ th iteration $(\boldsymbol{k} \geq \mathbf{1})$ : Compose the matrix $\Lambda^{(k-1)}$ from (25).

Solve Equation (26) to find $\boldsymbol{u}^{(k)}$.

Compute $\hat{b}_{m}^{(k)}$ and $\hat{g}_{m}^{(k)}$ from (27).

Stop iterating if $\boldsymbol{u}^{(k)}=\left(\begin{array}{llllll}1 & 1 & 1 & 0 & 0 & 0\end{array}\right)^{T}$ so that $\tilde{b}_{m}^{(k)}=0$ and $\tilde{g}_{m}^{(k)}=1$.

This algorithm actually always converges in at most two iterations. In fact, in the first iteration, the $n$th row of (26) is

$$
\sum_{m=1}^{3}\left(\frac{V_{m, n}-\hat{b}_{m}^{(0)}}{\hat{g}_{m}^{(0)}}\right)^{2} u_{m}^{(1)}+\frac{V_{m, n}-\hat{b}_{m}^{(0)}}{\hat{g}_{m}^{(0)}} w_{m}^{(1)}=1 .
$$

We insert $\hat{g}_{m}^{(0)}=\hat{g}_{m}^{(1)} \sqrt{u_{m}^{(1)}}$ and $\hat{b}_{m}^{(0)}=\hat{b}_{m}^{(1)}+\frac{w_{m}^{(1)}}{2 u_{m}^{(1)}} \hat{g}_{m}^{(0)}$ from (27) into this equation, expand the first square and simplify to

$$
1=\sum_{m=1}^{3}\left(\frac{V_{m, n}-\hat{b}_{m}^{(1)}}{\hat{g}_{m}^{(1)}}\right)^{2} \frac{\operatorname{sgn}\left(u_{m}^{(1)}\right)}{1+\sum_{k=1}^{3} \frac{\left(w_{k}^{(1)}\right)^{2}}{4 u_{k}^{(1)}}}
$$

For invertible $\Lambda^{(1)}$, comparison with (26) for $k=2$ gives that

$$
\boldsymbol{u}^{(2)}=\left(\begin{array}{llllll}
u_{1}^{(2)} & u_{2}^{(2)} u_{3}^{(2)} & 0 & 0 & 0
\end{array}\right)^{T} \quad \text { with } \quad u_{m}^{(2)}=\frac{\operatorname{sgn}\left(u_{m}^{(1)}\right)}{1+\sum_{k=1}^{3} \frac{\left(w_{k}^{(1)}\right)^{2}}{4 u_{k}^{(1)}}}
$$


Insertion of (29) in (27) gives

$$
\begin{aligned}
& \hat{b}_{m}^{(2)}=\hat{b}_{m}^{(1)}=\hat{b}_{m}^{(0)}-\frac{w_{m}^{(1)}}{2 u_{m}^{(1)}} \hat{g}_{m}^{(0)} \text { and } \\
& \hat{g}_{m}^{(2)}=\frac{\hat{g}_{m}^{(1)}}{\sqrt{u_{m}^{(2)}}}=\frac{\hat{g}_{m}^{(0)}}{\sqrt{u_{m}^{(1)}}} \sqrt{\frac{1+\sum_{k=1}^{3} \frac{\left(w_{k}^{(1)}\right)^{2}}{4 u_{k}^{(1)}}}{\operatorname{sgn}\left(u_{m}^{(1)}\right)}} .
\end{aligned}
$$

Consequently, insertion of (30) in (28) gives $1=\sum_{m=1}^{3}\left(\frac{V_{m, n}-\hat{b}_{m}^{(1)}}{\hat{g}_{m}^{(1)}}\right)^{2} u_{m}^{(2)}=\sum_{m=1}^{3}\left(\frac{V_{m, n}-\hat{b}_{m}^{(2)}}{\hat{g}_{m}^{(2)}}\right)^{2}$. By (26) $)$, this means that $\Lambda^{(2)} \boldsymbol{u}^{(3)}=\mathbf{1}$ with $\boldsymbol{u}^{(3)}=\left(\begin{array}{llllll}1 & 1 & 1 & 0 & 0 & 0\end{array}\right)^{T}$ so the algorithm has converged to the solution (30), which is identical to (7) in general and identical to (6) in the special case with all $b_{m}=0$ and $g_{m}=1$.

For this algorithm to work, we need the same requirements on invertibility of $\Lambda^{(k)}$ and on the signs of $\hat{g}_{m}^{(k)}, u_{m}^{(k)}$ that were needed and treated more carefully in Theorems 1 and 2 .

\section{Conclusions}

We have derived a simple non-iterative calibration method in Theorem 2. Its main advantages are that only from the accelerometer output voltages it gives a complete knowledge of whether it is possible, with any method, to recover the accelerometer parameters $b_{m}^{\text {true }}$ and $g_{m}^{\text {true }}$ from the output voltages, and when this is possible, we provide a simple explicit formula (6) for computing them with a smaller number of arithmetic operations than previous iterative approaches. We also show how the iterative method proposed in [6] is related to our method and shows that it always converges in at most two iterations.

We proved in Theorem 3 that $b_{m}^{\text {true }}$ and $g_{m}^{\text {true }}$ can be safely recovered for any deviations from the suggested 90 degree rotations with angles smaller than some upper bound. We provided an estimate $\alpha$ from below of this upper bound, and found that $\alpha=5^{\circ}$ for the Colibrys SF3000L accelerometers in our lab.

Our Monte Carlo simulations demonstrated a similar robustness against up to 10 degrees misalignment from the horizontal plane for noisy environments with cross-axis interference. They also indicated that getting closer to horizontal alignment with six calibration measurements can give give more robustness against cross-axis interference than trying to compensate for the 10 degrees misalignment with the investigated least squares approaches and up to 15 calibration measurements. For higher accuracy, a natural next step would be to generalize our method from the six parameter model (1c) to the related nine parameter model for nonorthogonal axes that is used, for example in 10.

For 90 degrees rotations and six measurements with $0.5 \%$ cross-axis interference, noise standard deviation 0.01 and the accelerometer parameters (14) used in our simulations, we got, for instance, less than $0.016 \%$ relative error in the gain estimates and errors in $b_{m}, g_{m}$ of size corresponding roughly to a fourth decimal error in measurements of the Earth gravity.

\section{A Proof of Theorem 1}

We will prove the equivalence by establishing an invertible mapping of the set of all $\boldsymbol{u}$ satisfying (44) onto the set of all $\boldsymbol{b}, \boldsymbol{g}$ satisfying (5). This shows both that uniqueness is preserved 
(both sets contain the same number of elements) and that the conditions (4) via the established mapping are equivalent to the conditions (5).

Hence, first assume that (44) holds. We can then write the equation $V^{+} \boldsymbol{u}=\mathbf{1}$ in the form

$$
V_{1, n}^{2} u_{1}+V_{1, n} w_{1}+V_{2, n}^{2} u_{2}+V_{2, n} w_{2}+V_{3, n}^{2} u_{1}+V_{3, n} w_{1}=1 \quad \text { for } n=1, \ldots, 6,
$$

which can be completed to three full squares by adding $\frac{w_{1}^{2}}{4 u_{1}}+\frac{w_{2}^{2}}{4 u_{2}}+\frac{w_{3}^{2}}{4 u_{3}}$, so that

$$
\sum_{m=1}^{3} u_{m}\left(V_{m, n}-\left(-\frac{w_{m}}{2 u_{m}}\right)\right)^{2}=1+\frac{w_{1}^{2}}{4 u_{1}}+\frac{w_{2}^{2}}{4 u_{2}}+\frac{w_{3}^{2}}{4 u_{3}} .
$$

Since all $u_{m} \neq 0$ and $\operatorname{sgn}\left(u_{m}\right)=\operatorname{sgn}\left(1+\sum_{k=1}^{3} \frac{w_{k}^{2}}{4 u_{k}}\right)$ we can let $b_{m}$ and positive $g_{m}$ be defined by (6) and rewrite (32) in the form $\sum_{m=1}^{3} \frac{\left(V_{m, n}-b_{m}\right)^{2}}{g_{m}{ }^{2}}=1$. It also follows that

$$
\sum_{m=1}^{3} \frac{b_{m}^{2}}{g_{m}^{2}}=\sum_{m=1}^{3} \frac{\frac{w_{m}^{2}}{4 u_{m}}}{1+\sum_{k=1}^{3} \frac{w_{k}^{2}}{4 u_{k}}}=\frac{\sum_{m=1}^{3} \frac{w_{m}^{2}}{4 u_{m}}}{1+\sum_{k=1}^{3} \frac{w_{k}^{2}}{4 u_{k}}} \neq 1
$$

Consequently, (6) defines a function $f$ that maps $\boldsymbol{u}$ satisfying (4) to $(\boldsymbol{b}, \boldsymbol{g}$ ) satisfying (5). To show that $f$ is invertible, we choose arbitrary $\boldsymbol{b}, \boldsymbol{g}$ satisfying (5), for which we need to find a unique $\boldsymbol{u}$ satisfying (4) and such that $f(\boldsymbol{u})=(\boldsymbol{b}, \boldsymbol{g})$. In other words, we need to find a unique solution to the equations $w_{m}=-2 b_{m} u_{m}$ and $g_{m}^{2}=\frac{1+\sum_{k=1}^{3} b_{k}^{2} u_{k}}{u_{m}}$, which we can rewrite as a system of linear equations

$$
\left(\begin{array}{ccc}
g_{1}^{2}-b_{1}^{2} & -b_{2}^{2} & -b_{3}^{2} \\
-b_{1}^{2} & g_{2}^{2}-b_{2}^{2} & -b_{3}^{2} \\
-b_{1}^{2} & -b_{2}^{2} & g_{3}^{2}-b_{3}^{2}
\end{array}\right)\left(\begin{array}{l}
u_{1} \\
u_{2} \\
u_{3}
\end{array}\right)=\left(\begin{array}{l}
1 \\
1 \\
1
\end{array}\right)
$$

which has exactly the same solutions as

$$
\left(\begin{array}{ccc}
g_{1}^{2} & 0 & -g_{3}^{2} \\
0 & g_{2}^{2} & -g_{3}^{2} \\
0 & 0 & g_{3}^{2}\left(1-\sum_{k=1}^{3} \frac{b_{k}^{2}}{g_{k}^{2}}\right)
\end{array}\right)\left(\begin{array}{l}
u_{1} \\
u_{2} \\
u_{3}
\end{array}\right)=\left(\begin{array}{l}
0 \\
0 \\
1
\end{array}\right)
$$

Since all $g_{m}>0$ and $\sum_{k=1}^{3} \frac{b_{k}^{2}}{g_{k}^{2}} \neq 1$ by (5) , there is exactly one solution of (33), namely

$$
u_{m}=\frac{1}{g_{m}^{2}\left(1-\sum_{k=1}^{3} \frac{b_{k}^{2}}{g_{k}^{2}}\right)} \quad \text { and } \quad w_{m}=-2 b_{m} u_{m}=-\frac{2 b_{m}}{g_{m}^{2}\left(1-\sum_{k=1}^{3} \frac{b_{k}^{2}}{g_{k}^{2}}\right)},
$$

from which it also follows that $u_{m} \neq 0, \operatorname{sgn}\left(u_{m}\right)=\operatorname{sgn}\left(1-\sum_{k=1}^{3} \frac{b_{k}^{2}}{g_{k}^{2}}\right)$ and

$$
\begin{aligned}
\operatorname{sgn}\left(1+\sum_{k=1}^{3} \frac{w_{k}^{2}}{4 u_{k}}\right) & =\operatorname{sgn}\left(1+\sum_{k=1}^{3} b_{k}^{2} u_{k}\right)=\operatorname{sgn}\left(1+\sum_{k=1}^{3} \frac{b_{k}^{2}}{g_{k}^{2}\left(1-\sum_{i=1}^{3} \frac{b_{i}^{2}}{g_{i}^{2}}\right)}\right) \\
& =\operatorname{sgn}\left(\frac{1}{1-\sum_{k=1}^{3} \frac{b_{k}^{2}}{g_{k}^{2}}}\right)=\operatorname{sgn}\left(1-\sum_{k=1}^{3} \frac{b_{k}^{2}}{g_{k}^{2}}\right)=\operatorname{sgn}\left(u_{m}\right) .
\end{aligned}
$$

Thus, insertion of (6) in $\sum_{m=1}^{3} \frac{\left(V_{m, n}-b_{m}\right)^{2}}{g_{m}^{2}}=1$ gives (32), which implies (31). Hence, we have found a unique $\boldsymbol{u}$ satisfying (44) such that $f(\boldsymbol{u})=(\boldsymbol{b}, \boldsymbol{g})$, which completes the proof. 


\section{B Proof of Theorem 2}

Firstly, assume that $V^{+} \boldsymbol{u}=\mathbf{1}$ has a unique solution. From $\sum_{m=1}^{3}\left(\frac{V_{m, n}-b_{m}}{g_{m}}\right)^{2}=1$ we have $\sum_{m=1}^{3} \frac{V_{m, n}^{2}}{g_{m}^{2}}-\frac{2 b_{m} V_{m, n}}{g_{m}^{2}}=1-\sum_{k=1}^{3} \frac{b_{k}^{2}}{g_{k}^{2}}$, which for $\sum_{k=1}^{3} \frac{b_{k}^{2}}{g_{k}^{2}} \neq 1$ implies

$$
\sum_{m=1}^{3} \frac{V_{m, n}^{2}}{g_{m}^{2}\left(1-\sum_{k=1}^{3} \frac{b_{k}^{2}}{g_{k}^{2}}\right)}-\frac{2 b_{m} V_{m, n}}{g_{m}^{2}\left(1-\sum_{k=1}^{3} \frac{b_{k}^{2}}{g_{k}^{2}}\right)}=1
$$

Thus, $\boldsymbol{u}$, where $u_{m}=\frac{1}{g_{m}^{2}\left(1-\sum_{k=1}^{3} \frac{b_{k}^{2}}{g_{k}^{2}}\right)}$ and $w_{m}=-\frac{2 b_{m}}{g_{m}^{2}\left(1-\sum_{k=1}^{3} \frac{b_{k}^{2}}{g_{k}^{2}}\right)}$ is a solution of $V^{+} \boldsymbol{u}=\mathbf{1}$ and by the assumption above it is the only solution. Moreover, for $\sum_{k=1}^{3} \frac{b_{k}^{2}}{g_{k}^{2}} \neq 1$ we have $u_{m} \neq 0$ and, by (35) $\operatorname{sgn}\left(u_{m}\right)=\operatorname{sgn}\left(1+\sum_{k=1}^{3} \frac{w_{k}^{2}}{4 u_{k}}\right)$ for $m=1,2,3$. Thus, the sufficient condition of Theorem 1 is satisfied and we get that $\boldsymbol{b}$ and $\boldsymbol{g}$ are unique.

The inverse statement follows directly from Theorem 1 .

\section{Proof of Theorem 3}

We begin with a few preliminaries needed for this.

For a matrix $M$, we write $M^{-1}$ for the inverse of $M$. The $\infty$-norm for an $m \times n$ matrix $M$ is

$$
\|M\|_{\infty}=\max _{1 \leq k \leq m} \sum_{l=1}^{n}\left|M_{k, l}\right| .
$$

It shares the submultiplicative property with other operator norms, that is, for all $n \times n$-matrices $A, B$ [11, equation (2.3.4)]

$$
\|A B\|_{\infty} \leq\|A\|_{\infty}\|B\|_{\infty} .
$$

The following theorem is a special case of the more general result [11, Theorem 2.3.4].

Theorem 4. If $\Lambda$ is invertible and $\left\|\Lambda^{-1} E\right\|_{\infty}<1$, then $\Lambda+E$ is invertible.

Proof of Theorem 3. The condition $\frac{\left|b_{m}\right|}{g_{m}} \leq \beta \leq \frac{1}{2}$ implies that $S \leq 3 / 4<1$. Moreover, for $\mathcal{A}_{m}^{ \pm} \stackrel{\text { def }}{=} \frac{S-1 \pm \frac{b_{m}}{g_{m}}-\frac{b_{m}^{2}}{g_{m}^{2}}}{2 g_{m}^{2}}, \mathcal{B}_{m, n}^{ \pm} \stackrel{\text { def }}{=}-\frac{b_{n}\left(b_{n} \pm g_{n}\right)}{2 g_{m}^{2} g_{n}^{2}}, \mathcal{C}_{m}^{ \pm} \stackrel{\text { def }}{=}-\frac{(S-1)\left(b_{m} \pm \frac{g_{m}}{2}\right) \mp \frac{b_{m}^{2}}{g_{m}}-\frac{b_{m}^{3}}{g_{m}^{2}}}{g_{m}^{2}}, \mathcal{D}_{m, n}^{ \pm} \stackrel{\text { def }}{=}-2 b_{m} \mathcal{B}_{m, n}^{ \pm}$and

$$
M \stackrel{\text { def }}{=}\left(\begin{array}{cccccc}
\mathcal{A}_{1}^{+} & \mathcal{B}_{1,2}^{-} & \mathcal{B}_{1,3}^{-} & \mathcal{A}_{1}^{-} & \mathcal{B}_{1,2}^{+} & \mathcal{B}_{1,3}^{+} \\
\mathcal{B}_{2,1}^{-} & \mathcal{A}_{2}^{+} & \mathcal{B}_{2,3}^{-} & \mathcal{B}_{2,1}^{+} & \mathcal{A}_{2}^{-} & \mathcal{B}_{2,3}^{+} \\
\mathcal{B}_{3,1}^{-} & \mathcal{B}_{3,2}^{-} & \mathcal{A}_{3}^{+} & \mathcal{B}_{3,1}^{+} & \mathcal{B}_{3,2}^{+} & \mathcal{A}_{3}^{-} \\
\mathcal{C}_{1}^{-} & \mathcal{D}_{1,2}^{-} & \mathcal{D}_{1,3}^{-} & \mathcal{C}_{1}^{+} & \mathcal{D}_{1,2}^{+} & \mathcal{D}_{1,3}^{+} \\
\mathcal{D}_{2,1}^{-} & \mathcal{C}_{2}^{-} & \mathcal{D}_{2,3}^{-} & \mathcal{D}_{2,1}^{+} & \mathcal{C}_{2}^{+} & \mathcal{D}_{2,3}^{+} \\
\mathcal{D}_{3,1}^{-} & \mathcal{D}_{3,2}^{-} & \mathcal{C}_{3}^{-} & \mathcal{D}_{3,1}^{+} & \mathcal{D}_{3,2}^{+} & \mathcal{C}_{3}^{+}
\end{array}\right)
$$

one can check, for example with Maple, that $\frac{1}{S-1} M V_{A_{0}, \boldsymbol{b}, \boldsymbol{g}}^{+}=V_{A_{0}, \boldsymbol{b}, \boldsymbol{g}}^{+} \frac{1}{S-1} M=I_{6 \times 6}$, so that $V_{A_{0}, \boldsymbol{b}, \boldsymbol{g}}^{+}$ is invertible and $V_{A_{0}, \boldsymbol{b}, \boldsymbol{g}}^{+}{ }^{-1}=\frac{1}{S-1} M$. Hence, for $E \stackrel{\text { def }}{=} V_{A, \boldsymbol{b}, \boldsymbol{g}}^{+}-V_{A_{0}, \boldsymbol{b}, \boldsymbol{g}}^{+}$, Theorem 4 tells that if $\left\|V_{A_{0}, \boldsymbol{b}, \boldsymbol{g}}^{+}{ }^{-1} E\right\|_{\infty}<1$, then $V_{A_{0}, \boldsymbol{b}, \boldsymbol{g}}^{+}+E=V_{A, \boldsymbol{b}, \boldsymbol{g}}^{+}$is invertible. Thus we can prove this theorem in four steps: Step 1: Estimate $\left\|V_{A_{0}, \boldsymbol{b}, \boldsymbol{g}}^{+1}\right\|_{\infty}$; Step 2: Estimate $\|E\|_{\infty}$; Step 3: Show that (19) 
implies that $\left\|V_{A_{0}, \boldsymbol{b}, \boldsymbol{g}}^{+1} E\right\|_{\infty} \leq\left\|V_{A_{0}, \boldsymbol{b}, \boldsymbol{g}}^{+1}\right\|_{\infty}\|E\|_{\infty}<1$, so that $V_{A_{0}, \boldsymbol{b}, \boldsymbol{g}}^{+}+E=V_{A, \boldsymbol{b}, \boldsymbol{g}}^{+}$is invertible; Step 4: Prove the last statement of the theorem.

Step 1: For $k=1, \ldots, 6$ and $m=4,5,6$, (17) gives that

$$
E_{k, m} \stackrel{\text { def }}{=}\left(V_{A, \boldsymbol{b}, \boldsymbol{g}}^{+}-V_{A_{0}, \boldsymbol{b}, \boldsymbol{g}}^{+}\right)_{k, m}=g_{m-3}\left(\mathcal{A}_{m-3, k}-\left(A_{0}\right)_{m-3, k}\right)=g_{m-3} \varepsilon_{m-3, k} .
$$

Similarly, for $k=1, \ldots, 6$ and $m=1,2,3$,

$$
\begin{aligned}
E_{k, m} & =\left(V_{A, \boldsymbol{b}, \boldsymbol{g}}\right)_{m, k}^{2}-\left(V_{A_{0}, \boldsymbol{b}, \boldsymbol{g}}\right)_{m, k}^{2}=\left(\mathcal{A}_{m, k} g_{m}+b_{m}\right)^{2}-\left(\left(A_{0}\right)_{m, k} g_{m}+b_{m}\right)^{2} \\
& =\left(\mathcal{A}_{m, k}^{2}-\left(A_{0}\right)_{m, k}^{2}\right) g_{m}^{2}+2\left(\mathcal{A}_{m, k}-\left(A_{0}\right)_{m, k}\right) g_{m} b_{m} \\
& =\left(A-A_{0}\right)_{m, k}\left(A+A_{0}\right)_{m, k} g_{m}^{2}+2 \varepsilon_{m, k} g_{m} b_{m} \\
& =\varepsilon_{m, k}\left(\varepsilon_{m, k}+2\left(A_{0}\right)_{m, k}\right) g_{m}^{2}+2 \varepsilon_{m, k} g_{m} b_{m} \\
& =g_{m}^{2} \varepsilon_{m, k}^{2}+2 g_{m}\left(b_{m}+\left(A_{0}\right)_{m, k} g_{m}\right) \varepsilon_{m, k} \\
& = \begin{cases}g_{m}^{2} \varepsilon_{m, k}^{2}+2 g_{m}\left(b_{m}+g_{m}\right) \varepsilon_{m, k}, & \text { if } k=m, \\
g_{m}^{2} \varepsilon_{m, k}^{2}+2 g_{m}\left(b_{m}-g_{m}\right) \varepsilon_{m, k}, & \text { if } k=m+3, \\
g_{m}^{2} \varepsilon_{m, k}^{2}+2 g_{m} b_{m} \varepsilon_{m, k}, & \text { otherwise. }\end{cases}
\end{aligned}
$$

By (36) and the assumptions of this theorem, we now get

$$
\begin{aligned}
\|E\|_{\infty} & \leq \varepsilon^{2}\left(\sum_{m=1}^{3} g_{m}^{2}\right)+2 \varepsilon\left(\sum_{m=1}^{3}\left|b_{m}\right| g_{m}\right)+2 \varepsilon \gamma^{2}+\varepsilon \sum_{m=1}^{3} g_{m} \\
& \leq 3 \gamma^{2} \varepsilon^{2}+\left(6 \beta \gamma^{2}+2 \gamma^{2}+3 \gamma\right) \varepsilon=\gamma^{2}\left(3 \varepsilon^{2}+\left(6 \beta+2+\frac{3}{\gamma}\right) \varepsilon\right) .
\end{aligned}
$$

Step 2: For $i=1,2,3$,

$$
\sum_{j=1}^{6}\left|M_{i, j}\right|=\left|\mathcal{A}_{i}^{+}\right|+\left|\mathcal{A}_{i}^{-}\right|+\sum_{j=1}^{3}\left(\left|\mathcal{B}_{i, j}^{-}\right|+\left|\mathcal{B}_{i, j}^{+}\right|\right)-\left|\mathcal{B}_{i, i}^{-}\right|-\left|\mathcal{B}_{i, i}^{+}\right|
$$

For $\frac{\left|b_{i}\right|}{g_{i}} \leq \beta \leq \frac{1}{2}$ it follows that $1-S \pm \frac{b_{i}}{g_{i}}+\frac{b_{i}^{2}}{g_{i}^{2}} \geq 1-S+\frac{b_{i}^{2}}{g_{i}^{2}}-\frac{\left|b_{i}\right|}{g_{i}} \geq 1-\frac{2}{4}-\frac{1}{2}=0$, so that

$$
\begin{aligned}
\sum_{j=1}^{6}\left|M_{i, j}\right|= & \frac{1-S-\frac{b_{i}}{g_{i}}+\frac{b_{i}^{2}}{g_{i}^{2}}}{2 g_{i}^{2}}+\frac{1-S+\frac{b_{i}}{g_{i}}+\frac{b_{i}^{2}}{g_{i}^{2}}}{2 g_{i}^{2}} \\
& +\sum_{j=1}^{3}\left(\frac{\left|b_{j}\right|\left(g_{j}-b_{j}\right)}{2 g_{i}^{2} g_{j}^{2}}+\frac{\left|b_{j}\right|\left(b_{j}+g_{j}\right)}{2 g_{i}^{2} g_{j}^{2}}\right)-\frac{\left|b_{i}\right|\left(g_{i}-b_{i}\right)}{2 g_{i}^{2} g_{i}^{2}}-\frac{\left|b_{i}\right|\left(g_{i}+b_{i}\right)}{2 g_{i}^{2} g_{i}^{2}} \\
= & \frac{1}{g_{i}^{2}}\left(1-S+\frac{b_{i}^{2}}{g_{i}^{2}}\right)+\sum_{j=1}^{3}\left(\frac{\left|b_{j}\right|}{g_{i}^{2} g_{j}}\right)-\frac{\left|b_{i}\right|}{g_{i}^{2} g_{i}}=\frac{1}{g_{i}^{2}}\left(1-S+\frac{b_{i}^{2}}{g_{i}^{2}}-\frac{\left|b_{i}\right|}{g_{i}}+\sum_{j=1}^{3} \frac{\left|b_{j}\right|}{g_{j}}\right) \\
\leq & \frac{1}{g_{i}^{2}}\left(1-S+\sum_{j=1}^{3} \frac{\left|b_{j}\right|}{g_{j}}\right) .
\end{aligned}
$$


For $\sigma \stackrel{\text { def }}{=} \min g_{m},\left|b_{m}\right| / g_{m} \leq \beta \leq 1 / 2$ and $i=1,2,3$ we obtain

$$
\begin{aligned}
\sum_{j=1}^{6}\left|M_{i, j}\right| & \leq \frac{1}{\sigma^{2}}\left(1-S+\sum_{j=1}^{3} \frac{\left|b_{j}\right|}{g_{j}}\right)=\frac{1}{\sigma^{2}}\left(\frac{7}{4}-\sum_{j=1}^{3}\left(\frac{1}{2}-\frac{\left|b_{j}\right|}{g_{j}}\right)^{2}\right) \\
& \leq \frac{1}{\sigma^{2}}\left(\frac{7}{4}-3\left(\frac{1}{2}-\beta\right)^{2}\right)=\frac{1}{\sigma^{2}}\left(1+3 \beta-3 \beta^{2}\right) .
\end{aligned}
$$

Similarly, for $i=4,5,6$ we have

$$
\sum_{j=1}^{6}\left|M_{i, j}\right|=\left|\mathcal{C}_{i-3}^{-}\right|+\left|\mathcal{C}_{i-3}^{-}\right|+\sum_{j=1}^{3}\left(\left|\mathcal{D}_{i-3, j}^{-}\right|+\left|\mathcal{D}_{i-3, j}^{+}\right|\right)-\left|\mathcal{D}_{i-3, i-3}^{-}\right|-\left|\mathcal{D}_{i-3, i-3}^{+}\right|
$$

For $\left|b_{m}\right| / g_{m} \leq \beta \leq 1 / 2$ we get $g_{m} / 2 \pm b_{m} \geq\left|b_{m}\right| \pm b_{m} \geq 0$ and $b_{m}^{2} / g_{m} \pm b_{m}^{3} / g_{m}^{2}=b_{m}^{2} / g_{m}(1 \pm$ $\left.b_{m} / g_{m}\right) \geq 0$. Consequently,

$$
\begin{aligned}
\mid(S-1)( & \left.b_{i-3} \mp \frac{g_{i-3}}{2}\right) \pm \frac{b_{i-3}^{2}}{g_{i-3}}-\frac{b_{i-3}^{3}}{g_{i-3}^{2}} \mid= \pm\left((S-1)\left(b_{i-3} \mp \frac{g_{i-3}}{2}\right) \pm \frac{b_{i-3}^{2}}{g_{i-3}}-\frac{b_{i-3}^{3}}{g_{i-3}^{2}}\right) \quad \text { and } \\
\sum_{j=1}^{6}\left|M_{i, j}\right|= & \frac{(S-1)\left(b_{i-3}-\frac{g_{i-3}}{2}\right)+\frac{b_{i-3}^{2}}{g_{i-3}}-\frac{b_{i-3}^{3}}{g_{i-3}^{2}}-\frac{(S-1)\left(b_{i-3}+\frac{g_{i-3}}{2}\right)-\frac{b_{i-3}^{2}}{g_{i-3}}-\frac{b_{i-3}^{3}}{g_{i-3}^{2}}}{g_{i-3}^{2}}}{}+\sum_{j=1}^{3}\left(\frac{\left|b_{j} b_{i-3}\right|\left(g_{j}-b_{j}\right)}{g_{j}^{2} g_{i-3}^{2}}+\frac{\left|b_{j} b_{i-3}\right|\left(b_{j}+g_{j}\right)}{g_{j}^{2} g_{i-3}^{2}}\right) \\
& -\frac{\left|b_{i-3} b_{i-3}\right|\left(g_{i-3}-b_{i-3}\right)}{g_{i-3}^{2} g_{i-3}^{2}}-\frac{\left|b_{i-3} b_{i-3}\right|\left(g_{i-3}+b_{j}\right)}{g_{i-3}^{2} g_{i-3}^{2}} \\
= & \frac{1}{g_{i-3}^{2}}\left(g_{i-3}(1-S)+\frac{2\left|b_{i-3}\right|^{2}}{g_{i-3}}\right)+\sum_{j=1}^{3}\left(\frac{2\left|b_{j} b_{i-3}\right|}{g_{i-3}^{2} g_{j}}\right)-\frac{2 b_{i-3}^{2}}{g_{i-3}^{2} g_{i-3}} \\
= & \frac{1}{g_{i-3}^{2}}\left(g_{i-3}(1-S)+2\left|b_{i-3}\right| \sum_{j=1}^{3}\left(\frac{\left|b_{j}\right|}{g_{j}}\right)\right) \\
\leq & \frac{1}{\sigma^{2}}\left(\gamma(1-S)+2 \beta \gamma \sum_{j=1}^{3}\left(\frac{\left|b_{j}\right|}{g_{j}}\right)\right)=\frac{\gamma}{\sigma^{2}}\left(1-\left(S-2 \beta \sum_{j=1}^{3}\left(\frac{\left|b_{j}\right|}{g_{j}}\right)+3 \beta^{2}\right)+3 \beta^{2}\right) \\
= & \left.\frac{\gamma}{\sigma^{2}}\left(1+3 \beta^{2}-\sum_{j=1}^{3}\left(\beta-\frac{\left|b_{j}\right|}{g_{j}}\right)\right)^{2}\right) \leq \frac{1}{\sigma^{2}} \gamma\left(1+3 \beta^{2}\right) .
\end{aligned}
$$

Thus, $\|M\|_{\infty} \leq \frac{1}{\sigma^{2}} \max \left\{1+3 \beta-3 \beta^{2}, \gamma\left(1+3 \beta^{2}\right)\right\} \stackrel{\text { def }}{=} \frac{\Omega}{\sigma^{2}}$ and

$$
\left\|V_{A_{0}, \boldsymbol{b}, \boldsymbol{g}}^{+-1}\right\|_{\infty}=\frac{\|M\|_{\infty}}{1-S} \leq \frac{\Omega}{\sigma^{2}(1-S)} \leq \frac{\Omega}{\sigma^{2}\left(1-3 \beta^{2}\right)} .
$$

Step 3: By the inequalities (37) and (38) we now get $\|E\|_{\infty}\left\|V_{A_{0}, \boldsymbol{b}, \boldsymbol{g}}^{+}{ }^{-1}\right\|_{\infty}<1$ for $\varepsilon$ satisfying

$$
\begin{gathered}
\|E\|_{\infty} \leq \gamma^{2}\left(3 \varepsilon^{2}+\left(6 \beta+2+\frac{3}{\gamma}\right) \varepsilon\right)<\frac{\sigma^{2}\left(1-3 \beta^{2}\right)}{\Omega} \leq \frac{1}{\left\|V_{A_{0}, \boldsymbol{b}, \boldsymbol{g}}^{+}\right\|_{\infty}} \\
3 \varepsilon^{2}+\left(6 \beta+2+\frac{3}{\gamma}\right) \varepsilon<\frac{\sigma^{2}\left(1-3 \beta^{2}\right)}{\gamma^{2} \Omega}, \quad \varepsilon>0 .
\end{gathered}
$$


with solution (19).

Step 4: Now it only remains to prove the last statement. For each column vector $\boldsymbol{a}_{\mathbf{0}}$ in $A_{0}$ and corresponding column vector $\boldsymbol{a}$ in $A$, it is not difficult to check that $\left\|\boldsymbol{a}-\boldsymbol{a}_{\mathbf{0}}\right\|_{\infty} \stackrel{\text { def }}{=}$ $\max _{k}\left|\boldsymbol{a}-\boldsymbol{a}_{\mathbf{0}}\right|_{k} \leq\left\|\boldsymbol{a}-\boldsymbol{a}_{\mathbf{0}}\right\|_{2}$. Hence, (18) holds if, for all column vectors, $\left\|\boldsymbol{a}-\boldsymbol{a}_{\mathbf{0}}\right\|_{2} \leq \varepsilon$. Since the lengths $\|\boldsymbol{a}\|_{2}=\left\|\boldsymbol{a}_{\mathbf{0}}\right\|_{2}=1$, the angle $\alpha$ between $\boldsymbol{a}_{\mathbf{0}}$ and $\boldsymbol{a}$ then satisfies $\sin (\alpha / 2)=$ $\left\|\boldsymbol{a}-\boldsymbol{a}_{\mathbf{0}}\right\|_{2} / 2 \leq \varepsilon / 2$ with $0 \leq \alpha \leq \pi$. Thus $\alpha=2 \sin ^{-1}(\varepsilon / 2)$.

\section{References}

[1] M El-Diasty, A El-Rabbany, and S Pagiatakis. Temperature variation effects on stochastic characteristics for low-cost MEMS-based inertial sensor error. Meas. Sci. Technol., 18(11):3321-3328, June 2007. DOI: 10.1088/0957-0233/18/11/009

[2] Alan Lai, Daniel A. James, Jason P. Hayes, and Erol C. Harvey. Semi-automatic calibration technique using six inertial frames of reference. In Proc. SPIE, volume 5274, pages 531-542, 2004. DOI: $10.1117 / 12.530199$.

[3] J.C. Lötters, J. Schipper, P.H. Veltink, W. Olthuis, and P. Bergveld. Procedure for inuse calibration of triaxial accelerometers in medical applications. Sensor Actuat A: Phys, 68(1-3):221-228, June 1998. DOI: 10.1016/S0924-4247(98)00049-1.

[4] Paul Lukowicz, Holger Junker, and Gerhard Tröster. Automatic calibration of body worn acceleration sensors. Lect. Notes Comput. Sc., 3001:176-181, April 2004. DOI: 10.1007/978-3-540-24646-6_11.

[5] Albert Krohn, Michael Beigl, Christian Decker, Uwe Kochendörfer, Philip Robinson, and Tobias Zimmer. Inexpensive and automatic calibration for acceleration sensors. Lect. Notes Comput. Sc., 3598:245-258, April 2005. DOI: 10.1007/11526858_19.

[6] Seong-hoon Peter Won and Farid Golnaraghi. A triaxial accelerometer calibration method using a mathematical model. IEEE T Instrum Meas, 59(8):2144-2153, August 2010. DOI: 10.1109/TIM.2009.2031849.

[7] Z.C. Wu, Z.F. Wang, and Y. Ge. Gravity based online calibration for monolithic triaxial accelerometers' gain and offset drift. In Proceedings of the 4 th World Congress on Intelligent Control and Automation, volume 3, pages 2171-2175, Shanghai, P.R.China, 2002. DOI: $10.1109 /$ WCICA.2002.1021471.

[8] Iuri Frosio, Federico Pedersini, and N. Alberto Borghese. Autocalibration of MEMS accelerometers. IEEE T Instrum Meas, 58(6):2034-2041, June 2009. DOI: 10.1109/TIM.2008.2006137.

[9] Walter Rudin. Real and Complex Analysis. McGraw-Hill, third edition, 1987.

[10] G. Panahandeh, I. Skog, and M. Jansson. Calibration of the accelerometer triad of an inertial measurement unit, Maximum Likelihood estimation and Cramér-Rao bound. In 2010 International Conference on Indoor Positioning and Indoor Navigation (IPIN), page (6 pp), September 2010. DOI: 10.1109/IPIN.2010.5646832, WWW: https://eeweb01.ee.kth.se/upload/publications/reports/2010/IR-EE-SB_2010_046.pdf

[11] Gene H. Golub and Chales F. van Loan. Matrix Computations. The Johns Hopkins University Press, third edition, 1996. 